\title{
The Lyman Continuum Escape Survey: Ionizing Radiation from [O III]-strong Sources at a Redshift of 3.1
}

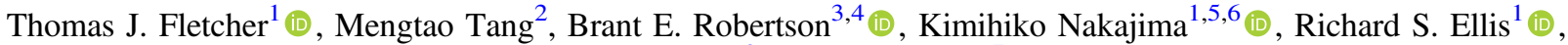 \\ Daniel P. Stark ${ }^{2}$, and Akio Inoue ${ }^{7}$ (i) \\ ${ }^{1}$ Department of Physics and Astronomy, University College London, Gower Street, London WC1E 6BT, UK \\ ${ }^{2}$ Steward Observatory, University of Arizona, 933 N. Cherry Ave., Tucson, AZ 85721, USA \\ ${ }^{3}$ Institute for Advanced Study, 1 Einstein Dr., Princeton, NJ 08540, USA \\ ${ }^{4}$ Department of Astronomy \& Astrophysics, University of California, Santa Cruz, 1156 High St., Santa Cruz, CA 95064, USA \\ ${ }^{5}$ European Southern Observatory (ESO), Karl-Schwarzschild-Strasse 2, D-85748 Garching, Germany \\ ${ }^{6}$ National Astronomical Observatory of Japan, 2-21-1 Osawa, Mitaka, Tokyo 181-8588, Japan \\ ${ }^{7}$ Osaka Sangyo University, 3-1-1 Nakagaito, Daito, Osaka 574-8530 Japan \\ Received 2018 June 5; revised 2019 May 1; accepted 2019 May 7; published 2019 June 18
}

\begin{abstract}
We present results from the LymAn Continuum Escape Survey (LACES), a Hubble Space Telescope (HST) program designed to characterize the ionizing radiation emerging from a sample of Ly $\alpha$-emitting galaxies at redshift $z \simeq 3.1$. As many show intense [O III] emission characteristic of $z>6.5$ star-forming galaxies, they may represent valuable low-redshift analogs of galaxies in the reionization era. Using HST Wide Field Camera 3/UVIS F336W to image Lyman continuum emission, we investigate the escape fraction of ionizing photons in this sample. For 61 sources, of which $77 \%$ are spectroscopically confirmed and 53 have measures of [O III] emission, we detect Lyman continuum leakage in $20 \%$, a rate significantly higher than is seen in individual continuum-selected Lyman break galaxies. We estimate that there is a $98 \%$ probability that $\leqslant 2$ of our detections could be affected by foreground contamination. Fitting multiband spectral energy distributions (SEDs) to take account of the varying stellar populations, dust extinctions and metallicities, we derive individual Lyman continuum escape fractions corrected for foreground intergalactic absorption. We find escape fractions of $15 \%-60 \%$ for individual objects and infer an average $20 \%$ escape fraction by fitting composite SEDs for our detected samples. Surprisingly, however, even a deep stack of those sources with no individual F336W detections provides a stringent upper limit on the average escape fraction of less than $0.5 \%$. We examine various correlations with source properties and discuss the implications in the context of the popular picture that cosmic reionization is driven by such compact, lowmetallicity star-forming galaxies.
\end{abstract}

Key words: early universe - galaxies: distances and redshifts - galaxies: evolution - galaxies: formation - galaxies: star formation - infrared: galaxies

\section{Introduction}

Deep imaging with the WFC3/IR camera on board Hubble Space Telescope (HST) has dramatically expanded our redshift horizon, making it practical to address two long-standing cosmological questions: (i) when did the universe transition from a neutral to an ionized state, and (ii) were early starforming galaxies responsible for this cosmic reionization? Multicolor HST/Spitzer imaging in the Ultra Deep Field (Beckwith et al. 2006; Ellis et al. 2013; Illingworth et al. 2013; Koekemoer et al. 2013) and the CANDELS fields (Grogin et al. 2011; Koekemoer et al. 2011; Oesch et al. 2013; Bouwens et al. 2015), together with complementary studies undertaken through the CLASH (Bradley et al. 2014) and Frontier Field (McLeod et al. 2015; Lotz et al. 2017) lensing clusters, have delivered several hundred $z>7$ Lyman break galaxy (LBG) candidates, providing the first convincing description of the abundance and luminosity distribution (McLure et al. 2013; Atek et al. 2015; Bouwens et al. 2015; Finkelstein et al. 2015; Livermore et al. 2017; Ishigaki et al. 2018) of early starforming galaxies to $z \simeq 10$ (see Stark 2016 for a review).

The optical depth, $\tau$, of electron scattering to the cosmic microwave background measured by the Planck consortium (Planck 2015) constrains the redshift window over which reionization occurred. Robertson et al. (2015) demonstrated how the demographics of star-forming galaxies determined by
$H S T$ can be reconciled with this value in terms of a reionization history over the redshift range $6 \lesssim z \lesssim 12$ given some significant assumptions about the ionizing capability of the typical, most abundant, low-luminosity sources. The key assumptions relate to (i) the UV radiation emerging from their stellar populations, defined by Robertson et al. (2013) in terms of $\xi_{\text {ion }}$, the number of Lyman continuum (LyC) photons produced per UV (1500 $⿱$ ) luminosity, and (ii) the fraction $f_{\text {esc }}$ of such LyC photons that can escape absorption within the galaxy and its immediate vicinity. The quantity $\xi_{\text {ion }}$ cannot be determined from broadband photometry alone (Robertson et al. 2013) and is best constrained from Balmer line emission using recombination physics with a weak dependence on $f_{\text {esc }}$ (Bouwens et al. 2016). Until James Webb Space Telescope is launched, the relevant lines are beyond reach of ground-based spectrographs at high redshift. Likewise, the opacity of the intergalactic medium (IGM) at UV wavelengths becomes too great beyond $z \simeq 4$ to determine $f_{\text {esc }}$ from deep HST imaging below the Lyman limit (e.g., Shapley et al. 2006). Therefore, neither of these quantities can be constrained for galaxies in the reionization era with current facilities, yet they collectively comprise the primary uncertainty in claims that reionization is driven by star-forming galaxies. The situation is particularly critical for $f_{\text {esc }}$ since Robertson et al. $(2013,2015)$ argued that a mean value of $10 \%-20 \%$ is required for galaxies to reionize the 
universe, whereas studies at redshifts where LyC photons can be directly detected frequently yield upper limits of $f_{\text {esc }} \lesssim 5 \%$ (e.g., Mostardi et al. 2015; Siana et al. 2015).

In promoting the view that early star-forming galaxies reionized the universe, many workers have speculated that both the intensity of the ionizing radiation (effectively $\xi_{\text {ion }}$ ) and the porosity of neutral gas in the circumgalactic medium (CGM; i.e., $f_{\text {esc }}$ ) increase with redshift, particularly for compact, intensely star-forming systems (Inoue et al. 2006; Kuhlen \& FaucherGiguère 2012; Robertson et al. 2013; Finkelstein et al. 2015). Indeed, the strength of nebular emission (e.g., [O III] $\lambda 5007$ ), whether measured directly from near-infrared spectroscopy (Schenker et al. 2013) or inferred indirectly from the excess flux in Spitzer photometry (Labbé et al. 2013; Smit et al. 2014, 2015), does apparently increase with redshift. Surprisingly, some of the most luminous LBGs with large $\left.\mathrm{EW}_{[\mathrm{O}} \mathrm{III}\right]$ at $z>7$ (Roberts-Borsani et al. 2016) also reveal Ly $\alpha$ in emission (Oesch et al. 2015; Zitrin et al. 2015; Laporte et al. 2017; Stark et al. 2017), "bucking the trend" established for less luminous systems. This correlation may imply that sources with large $\mathrm{EW}_{[\mathrm{O} \mathrm{III]}}$ also have a high value of $f_{\text {esc }}$, thereby creating early ionized bubbles that permit Ly $\alpha$ photons to emerge (Stark et al. 2017).

The interdependence of large $\mathrm{EW}_{[\mathrm{O} \text { III] }}$, a higher-than-average value of $\xi_{\text {ion }}$, and the leakage of LyC photons was first evaluated in the context of photoionization models by Nakajima \& Ouchi (2014). Compiling literature data, they found an interesting correlation between the emission-line ratio $[\mathrm{O}$ III] $] /[\mathrm{O}$ II $]$ (hereafter $\left.\mathrm{O}_{32}\right)$ and $f_{\text {esc }}$, which they claimed arises when $\mathrm{HII}$ regions are "density-bound" and some LyC leakage occurs. This picture contrasts with typical "ionization-bound" H II regions where LyC photons are fully absorbed within the radius of the associated Stromgren sphere. The conjecture has received further support by the recent detections of significant LyC radiation from nearby intense [O III] emitters (Izotov et al. 2016a, 2016b, 2018). The most extreme $\mathrm{O}_{32}$ sources in the Nakajima \& Ouchi (2014) study were narrowband-selected Ly $\alpha$ emitters (LAEs), whose observed properties are in many respects very similar to the dominant population of star-forming galaxies during reionization. Subsequently, through near-infrared and optical spectroscopy, Nakajima et al. $(2016,2018)$ provided further evidence that such LAEs have higher values of $\xi_{\text {ion }}$ than continuum-selected LBGs (Shivaei et al. 2018), and Tang et al. (2018) showed that $\xi_{\text {ion }}$ scales with [O III] $\mathrm{EW}$, reaching very large values in the most intense line emitters.

The most practical route to determine whether early galaxies reionized the IGM is to undertake a detailed study of analogs of this population at the highest redshift where direct measures of $\xi_{\text {ion }}$ and $f_{\text {esc }}$ are possible. With a representative sample of such analogs it may be possible to verify the inferred correlation between the [O III] emission and $f_{\text {esc }}$, as well as to determine the fraction of sources whose ionizing output (as defined by $\xi_{\text {ion }}$ and $f_{\text {esc }}$ ) would be sufficient if projected, into the $z>7$ population, to sustain reionization. Intermediate-redshift LAEs possibly represent the most valuable low-redshift analogs of the population of compact, low-mass, intensely star-forming galaxies that dominate the reionization era. Taking advantage of a large-area, narrowband-selected sample of spectroscopically confirmed LAEs in the SSA22 field (Hayashino et al. 2004; Matsuda et al. 2005; Yamada et al. 2012b; Nakajima et al. 2016, 2018), the LymAn Continuum Escape Survey (LACES) project aims to study these sources in detail and in particular to examine their LyC leakage via HST broadband imaging below the Lyman limit. A key question our survey can address is whether intense [O III] emission seen in many LAEs is associated with an increased $f_{\text {esc }}$ as conjectured originally by Nakajima \& Ouchi (2014). Deep UV imaging (and hence measures of $f_{\text {esc }}$ ) is presented for a unique and representative sample of $z \simeq 3.1$ LAE analogs for which the associated measures of Ly $\alpha$ and [O III] are already available from Keck and VLT spectroscopy.

A plan of the paper follows. In Section 2 we introduce our sample, which is based on HST imaging in three WFC3 fields spanning the area for which we have extensive ground-based optical and near-infrared spectroscopy. In this section we discuss the relevant imaging and spectroscopic data and their processing. In Section 3 we examine the new deep F336W images and devise a procedure for determining the presence of LyC leakage on a case-by-case basis in our sample, as well as the combined flux from those sources without individual detections. In Section 4 we define a path for deriving the measured $f_{\text {esc }}$ or limits on its value from the individual F336W fluxes, noting the dependences on the assumed form of the UV continuum as probed by independent spectroscopic measures

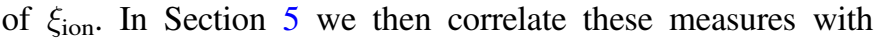
various source properties either measured observationally or derived from our model-dependent analyses. In Section 6, we discuss these correlations in the context of whether such [O III]-intense sources are likely prominent agents of cosmic reionization.

Throughout this paper, we adopt a concordance cosmology with $\Omega_{\Lambda}=0.7, \Omega_{m}=0.3$, and $H_{0}=70 \mathrm{~km} \mathrm{~s}^{-1} \mathrm{Mpc}^{-1}$. All magnitudes are given in the AB system (Oke \& Gunn 1983). When we refer to $f_{\text {esc }}$, we mean the absolute escape fraction of LyC photons unless specified otherwise.

\section{Data}

As discussed in Nakajima et al. (2016), our target sample is drawn from a Subaru imaging survey that identified $z \simeq 3.1$ LAEs in the SSA22 field (Hayashino et al. 2004; Matsuda et al. 2005; Yamada et al. 2012b) via their photometric excess in a narrowband filter at $497 \mathrm{~nm}$. In addition to initial spectroscopy to confirm their identity, we have undertaken a systematic campaign using optical spectrographs on Keck and the VLT to study their rest-frame UV emission lines (Nakajima et al. 2018) and near-infrared spectroscopy with Keck's MOSFIRE to examine their rest-frame optical emission, particularly the diagnostic lines of [O III] $\lambda 5007$ and [O II] $\lambda 3727$. Initial results from MOSFIRE were presented in Nakajima et al. (2016), but those data have been enlarged in the present paper to take account of the associated imaging data taken with $H S T$.

\subsection{Hubble Space Telescope Data}

The HST campaign (GO 14747, PI: Robertson) was conducted between UT 2017 May 14 and December 20, comprising four F160W pointings with WFC3/IR of one orbit each $(0.7 \mathrm{hr})$ and three WFC3/UVIS F336W pointings of 20 orbits each in five exposures per pointing (16 hr; see Table 1). Together with the narrowband images taken with Subaru, this strategy allows us to compare prospective Lyman continuum leakage in the F336W filter with associated signals in Ly $\alpha$ and the rest-frame optical continuum. 


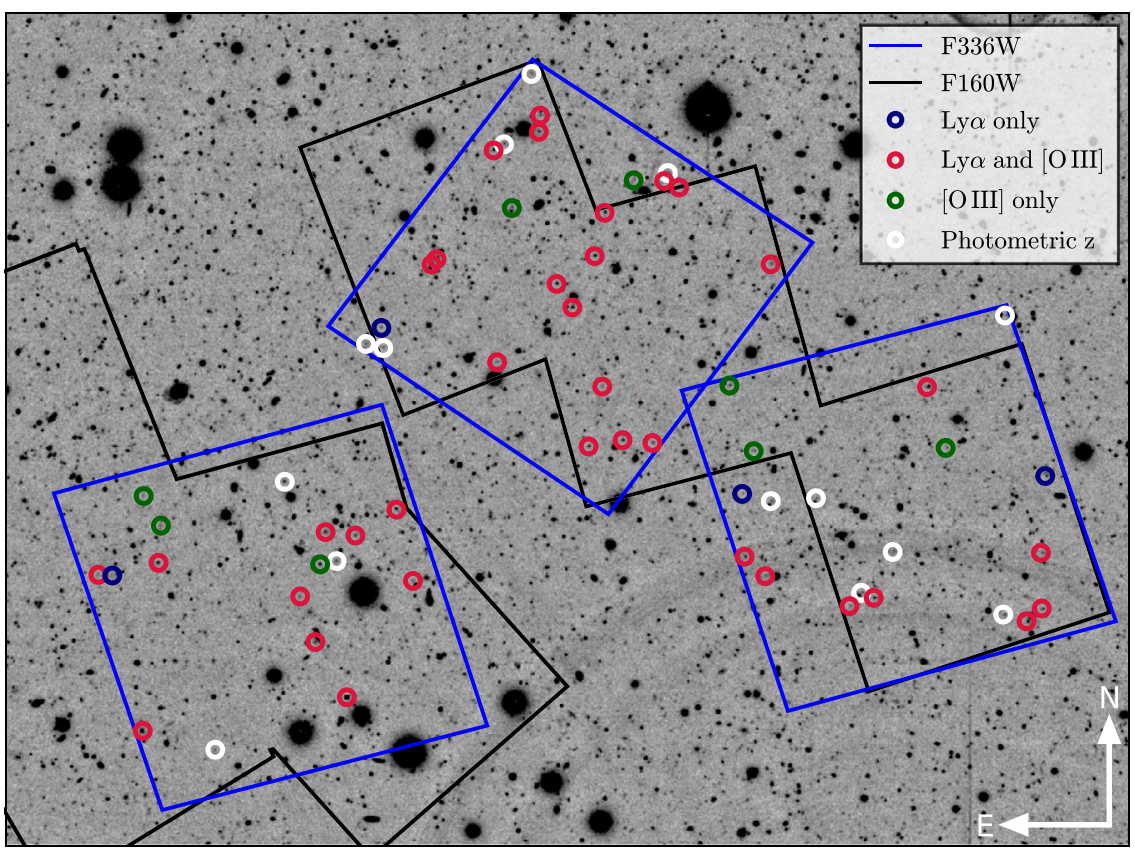

Figure 1. NB497 image showing the coverage of target Ly $\alpha$ emitters and LBGs from the full Subaru narrowband-selected sample within the three WFC3/UVIS F336W pointings. The area covered by the smaller WFC3/IR F160W pointings is delineated with overlapping black squares. The targets are color-coded according to their information content as follows: red for Ly $\alpha$ spectroscopic redshift and [O III] line flux, blue for Ly $\alpha$ spectroscopic redshift only, green for [O III] spectroscopic redshift only, and white for photometric data only. For detailed statistics see Table 3.

Table 1

HST Imaging Observations

\begin{tabular}{|c|c|c|c|c|c|c|}
\hline Filter & Pointing & $\begin{array}{l}\lambda_{\text {eff }} \\
(\AA)\end{array}$ & $\begin{array}{c}\mathrm{PSF}^{\mathrm{a}} \\
(\operatorname{arcsec})\end{array}$ & $\begin{array}{c}\text { Pixel } \\
\text { Scale } \\
\left(\operatorname{arcsec}_{\text {pixel }}{ }^{-1}\right)\end{array}$ & $\begin{array}{c}\text { Exposure } \\
\text { (s) }\end{array}$ & $\begin{array}{l}\text { Depth }^{\mathrm{b}} \\
(\mathrm{AB})\end{array}$ \\
\hline F336W & 1 & 3355 & 0.081 & 0.040 & 57845 & 30.24 \\
\hline F336W & 3 & 3355 & 0.081 & 0.040 & 57845 & 29.41 \\
\hline F160W & All & 15369 & 0.151 & 0.128 & 2612 & 27.61 \\
\hline
\end{tabular}

Notes.

a The FWHM of the PSF.

b The $3 \sigma$ limiting magnitude using an aperture with a diameter 1.5 times the size of the PSF FWHM (e.g., 0 .' 12 for the F336W images).

Within the coverage of the F336W imaging, there are 61 sources from the original Subaru sample, of which 54 are LAEs and 7 are LBGs. Although only 41 of the 54 LAEs have spectroscopic redshifts, we can exploit the remaining 13 narrowband-selected sources given that contamination from foreground emitters has been shown to be negligible in practice (Matsuda et al. 2005, 2006; Yamada et al. 2012a, 2012b). A summary of the statistical sample is given in Table 3, and their distribution in the three WFC3 fields is shown in Figure 1.

\subsubsection{WFC3/UVIS Reduction}

As described by Rafelski et al. (2015), the standard HST pipelines may not result in science images appropriate for the analysis of faint detections in WFC3/UVIS images. Correlated noise and other residual structure in the images can lead to the misidentification of artifacts as low signal-to-noise ratio $(\mathrm{S} / \mathrm{N})$ sources, which must be carefully addressed in any rigorous analysis. For the Hubble Deep UV Legacy Survey, Oesch et al. (2018) developed an approach to address the WFC3/UVIS data quality issue, described in their Section 4.4 and which we emulate here. The goal is to construct a sky dark frame from the dark-corrected F336W science exposures reduced using the STScI pipeline and then subtract the sky dark from each exposure to ameliorate the correlated noise and residual structure. First, sources were identified in the original science images resulting from the STScI pipeline by running Source Extractor (Bertin \& Arnouts 1996) with a detection threshold of $1.5 \sigma$ designed to capture genuine faint sources above typical noise fluctuations in the images. Based on the segmentation map of each pointing, all the pixels containing sources were masked and replaced by simulated noise computed using the average and standard deviation of background measured from nearby pixels around the sources. A background frame was thereby extracted from each of the 15 science exposures and then combined via median stacking to create the sky dark frame. The sky dark was subtracted from each of the 15 exposures, and these sky-darksubtracted frames were then combined into our final science images by creating median stacks for each of the three pointings using SWarp (Bertin 2010).

As an indication of the importance of our implementation of the additional sky dark subtraction, we compared our subsequent 
Table 2

Summary of Optical and NIR Imaging Data

\begin{tabular}{lcccc}
\hline \hline Filter & Observatory & $\begin{array}{c}\text { PSF } \\
(\operatorname{arcsec})^{\mathrm{a}}\end{array}$ & $\begin{array}{c}\text { Depth } \\
(\mathrm{mag})^{\mathrm{b}}\end{array}$ & Reference \\
\hline$u^{\star}$ & CFHT & 1.0 & 26.0 & $(1)$ \\
$B$ & Subaru & 1.0 & 26.5 & $(2),(3),(4)$ \\
$\mathrm{NB} 497$ & Subaru & 1.0 & 26.2 & $(2),(3),(4)$ \\
$V$ & Subaru & 1.0 & 26.6 & $(2),(3),(4)$ \\
$R$ & Subaru & 1.1 & 26.7 & $(2),(4)$ \\
$i^{\prime}$ & Subaru & 1.0 & 26.4 & $(2)$ \\
$z^{\prime}$ & Subaru & 1.0 & 25.7 & $(2)$ \\
$J$ & UKIRT & 0.9 & 23.5 & $(5)$ \\
$K$ & UKIRT & 0.8 & 23.1 & $(5)$ \\
{$[3.6]$} & Spitzer & 2.0 & $22.2-24.7$ & $(6)$ \\
{$[4.5]$} & Spitzer & 2.0 & $22.2-24.4$ & $(6)$ \\
\hline
\end{tabular}

Notes.

a The FWHM of the PSF.

$\mathrm{b}$ The $5 \sigma$ limiting magnitude using an aperture with a diameter of $3^{\prime \prime}$ for the IRAC 3.6 and $4.5 \mu \mathrm{m}$ bands and $2^{\prime \prime}$ for the other bands.

References (1) Hayashino et al. 2019; (2) Hayashino et al. 2004; (3) Yamada et al. 2012b; (4) Matsuda et al. 2005; (5) http://wsa.roe.ac.uk/; (6) http://sha. ipac.caltech.edu/applications/Spitzer/SHA/.

source identification and analysis with respect to one originally made using the STScI pipeline products. In addition to a significantly improved image quality, we find that our brightest F336W sources ( $\geqslant 4 \sigma$ significance) are robust to the choice of reduction, but the flux associated with these sources can vary substantially between reductions (from decreasing by $50 \%$ to increasing by $20 \%$ ). We further note that after the original submission of this paper, updated versions of the STScI science products became available (in 2019 January), correcting an issue whereby incorrect calibration products had been applied during the processing of WFC3/UVIS data for our program GO 14747. The final sky darks and science images used herein have been corrected for this issue, which also influenced the noise properties of the STScI products and the flux of objects measured in uncorrected data. We mention these issues to highlight the challenging systematics associated with analyzing WFC3/UVIS images at faint flux levels and to motivate our conservative sample definition where we restrict our Lyman continuum candidate designation to $\geqslant 4 \sigma \mathrm{F} 336 \mathrm{~W}$ sources.

To accurately compare F336W detections with signals in other bands, all HST images were astrometrically aligned with the most appropriate Subaru images using custom software and verified with visual inspection of hundreds of sources in each image. Sources were then extracted in the F336W, Subaru NB497 (Ly $\alpha$ ), and F160W images using a Python script based on the SEP tool ${ }^{8}$ (Bertin \& Arnouts 1996; Barbary 2016).

\subsection{Additional Photometry}

All the LACES targets are covered with the plentiful, deep multiwavelength photometric data in the SSA22 field. We utilize the photometric data, including Subaru, CFHT, UKIRT, and Spitzer/IRAC imaging data, in addition to the HST/ F160W photometry to constrain the nature of the stellar populations of the LACES objects via a spectral energy distribution (SED) fitting analysis (Section 4.2). Table 2 gives the details of the additional photometric data.

\footnotetext{
8 https://github.com/kbarbary/sep/tree/v1.0.x
}

We perform photometry of the bands listed in Table 2 on the LACES sources using TPHOT (v2.0; Merxlin et al. 2015 , 2016). Briefly, we use the $H S T / F 160 \mathrm{~W}$ image as a highresolution reference image and extract the spatial and morphological information of objects within a radius of $\sim 25^{\prime \prime}$ from each of the LACES sources. Using this information and a kernel carefully created to convolve the high-resolution image to have the point-spread function (PSF) of the lower-resolution ground-based images, TPHOT produces templates of the objects in the low-resolution image. TPHOT then varies the brightness of each of the templates to match the global observed flux in the low-resolution image. In this way we can accurately measure total fluxes from the low-resolution images in Table 2 by removing light from nearby contaminating sources. For sources not detected or not covered by the HST/ F160W image, we adopt aperture photometry with a $2^{\prime \prime}$ diameter aperture for the Subaru, CFHT, and UKIRT images and $3^{\prime \prime}$ for the IRAC data and fix the position of the aperture determined using the NB497 detection. The aperture magnitudes are then converted into total magnitudes using aperture correction values, which are estimated from differences between aperture and total magnitudes for point sources. We have confirmed that the two methods return a consistent SED within the $1 \sigma$ uncertainties for isolated objects.

\subsection{Near-infrared Spectroscopy}

In addition to the initial 2015 campaign reported in Nakajima et al. (2016), which targeted only one MOSFIRE pointing (referred to here as mask 1) in SSA22, we have now completed spectroscopy of three further pointings (masks 2-4) within the $H S T$-covered area (Figure 1). The new observations were taken on UT 2017 July 31, August 1, and October 10 in photometric conditions with seeing ranging from $0.5-0.9$ in the summer months to $0.3-0$ " 5 on the more recent run. Spectra were obtained in both the $K$ band (sampling [O III] and $\mathrm{H} \beta$ at a spectral resolution $R \simeq 3600$ ) and $H$ band (sampling [O II] at $R \simeq 3700$ ) for masks 1 and 2 , while only in the $K$ band for masks 3 and 4 . Individual exposures of $180 \mathrm{~s}(120 \mathrm{~s})$ were taken in $K(H)$ with an AB nod sequence of 3 !" 0 separation. The total on-source exposure times ranged from 2 to $3 \mathrm{hr}$, with some sources included on both mask 2 and mask 4 .

Data reduction was performed using the MOSFIRE DRP ${ }^{9}$ in the manner described in Nakajima et al. (2016). Briefly, the processing includes flat-fielding, wavelength calibration, background subtraction, and combining the nod positions. Wavelength calibration in $H$ was performed using $\mathrm{OH}$ sky lines, and in $K$ a combination of $\mathrm{OH}$ lines and neon arcs were used. Flux calibration and telluric absorption corrections were obtained from A0V Hipparcos stars observed at similar air masses, as well as via relatively bright stars $\left(K_{\mathrm{Vega}}=15.5-16.5\right)$ included on each of the masks.

We measured the [O II] and [O III] line fluxes by fitting a Gaussian profile to each line using the IRAF task spec fit. In deriving the rest-frame equivalent width (EW) of [O III], we used the measured F160W in conjunction with a mean SED of $z \simeq 3.1$ LAEs (Ono et al. 2010) to determine the continuum flux in the vicinity of the line near $2.05 \mu \mathrm{m}$. We investigated the effect of dust corrections using individually derived $E(B-V)$ values for each object (see Section 4), but as our LAEs are mostly dust-free, the corrections were small. Table 3 summarizes

\footnotetext{
9 https://keck-datareductionpipelines.github.io/MosfireDRP/
} 
Table 3

Summary of the LACES Sample

\begin{tabular}{lccc}
\hline \hline Number of Objects & LAEs & LBGs & Total \\
\hline Within the HST area & 54 & 7 & 61 \\
Spectroscopic redshift & 41 & 6 & 47 \\
F160W coverage or limits & 45 & 7 & 52 \\
[O III] or limits & 46 & 7 & 53 \\
[O II] or limits (with [O III] identified) & 23 & 4 & 27 \\
F160W coverage and both [O III]+[O II] data & 23 & 4 & 27 \\
\hline
\end{tabular}

the statistics of the [O II] and [O III] detections. The full catalog of line fluxes and EWs will be reported later as the spectroscopic campaign continues.

In total, 51 of the 61 sources in the three WFC3 fields have [O III] detections or upper limits. The coverage of [O II] is less complete at present, with roughly half of the [O III] sample containing [O II] data or upper limits (see Table 3).

\section{Lyman Continuum Candidates}

We now discuss the procedure adopted to decide which SSA22 sources show promising evidence of Lyman continuum leakage in the HST F336W filter. The key issues include the optimum aperture for measuring the $\mathrm{F} 336 \mathrm{~W}$ flux, the photometric significance of any detection, the spatial coincidence with signals in other bands, and the possibility of foreground contamination. We also discuss the nature of those sources where no significant F336W flux is seen and examine the possibility of providing a statistical detection on the basis of a stacking analysis.

\subsection{Detections}

We first constructed a mosaic of all 61 targets with $H S T$ F336W coverage, comparing the location and morphology of possible F336W detections with images in Subaru NB497 (Ly $\alpha), R$, and HST F160W. Five authors (M.T., B.E.R., T.F., R.S.E., D.P.S.) examined this mosaic for potential F336W detections. Although the Subaru Ly $\alpha$ image offers a natural astrometric reference point, as a ground-based image with 1 ." seeing it is less useful than the HST F160W image, which samples the rest-frame optical light and can reveal complex source morphology. In practice, we found it helpful to overlay a F160W contour over the F336W image to evaluate spatial coincidence.

The photometric significance of possible F336W detections was also taken into account on the assumption that LyC signals would be mostly unresolved with HST. Fluxes were measured in an aperture whose diameter is 1.5 times the $\mathrm{F} 336 \mathrm{~W}$ pointspread function (i.e., 3 WFC3/UVIS pixels, 0" $12,0.91 \mathrm{kpc}$ at $z=3.1$ ). This aperture is more sensitive in discovering candidates than adopting a (larger) matched aperture across all the photometric bands that would introduce unnecessary noise. For the few sources that show extended emission in the F336W image we only measure a signal from the brightest peak, possibly underestimating the true $\mathrm{F} 336 \mathrm{~W}$ flux and $f_{\text {esc }}$.

To evaluate the completeness of our search and provide useful upper limits for the nondetections, we masked all the detected sources above a threshold of $1.5 \sigma$ of the background noise. Fake sources with a Gaussian profile corresponding to the PSF of the images and known magnitudes were inserted into the unmasked regions and the detection algorithm rerun. In this manner we determined a $75 \%$ completeness limit of $\mathrm{F} 336 \mathrm{~W}(\mathrm{AB})=29.90$. We verified this noise limit with that determined from aperture measures in the vicinity of each target.

For each LyC candidate the noise level was measured locally in a $4 \times 4$ !. region around the target. The targets, neighboring objects, and any signal $5 \sigma$ above the noise level were masked in the postage stamps. Using these segmentation maps, apertures 1.5 times the PSF, the same size used to measure the flux, were randomly distributed and the $1 \sigma$ noise level calculated from the random placement of apertures on the image.

\subsection{Gold and Silver Subsamples}

We have conservatively divided our detections into Gold and Silver subsamples in order to distinguish between cases where we are respectively convinced and reasonably sure the detected LyC flux is associated with the target galaxy. In our subsequent analysis it will be helpful to examine trends separately between the Gold and Silver subsamples, as well as with those for the nondetections. We show in Figures 2 and 3 postage stamps of $4 \times 4$ ". for our Gold and Silver subsamples, defined according to the criteria below. Together they comprise 12 sources for which reasonably convincing F336W detections were found by the procedure outlined in Section 3.1. To assist in recognizing the detections, we also show the F336W images smoothed with a $2 \mathrm{D}$ Gaussian with $1 \sigma$ equal to 1 pixel. We also show an overlay of the F160W and Ly $\alpha$ narrowband contours on the F336W images to illustrate the spatial coincidence of the optical continuum and LyC flux. Table 4 summarizes the Gold and Silver subsamples alongside their photometric and spectroscopic properties.

To qualify for the Gold sample, targets must satisfy three criteria:

1. Availability of a spectroscopic redshift for the target or no evidence that the target may be an interloper. A redshift may seem an essential requirement, but the probability that an NB497 excess that leads to a selected $z=3.1$ LAE is contaminated by a foreground emission line is very low (Matsuda et al. 2005, 2006; Yamada et al. $2012 \mathrm{a}, 2012 \mathrm{~b}$ ), so only if there is some spectroscopic evidence for an interloper would the candidate be rejected.

2. The F336W flux must be spatially coincident (to within 0 ". 6) with the core of the F160W flux or the Ly $\alpha$ centroid. In cases where the F160W image reveals substructure, there is a danger that the F336W detection is coincident with an interloper. Although we will show that this possible contamination is unlikely, such a configuration merits demotion to the Silver subsample.

3. The F336W detection has $\mathrm{S} / \mathrm{N} \geqslant 4$ as evaluated by the process discussed in the next subsection.

Using these criteria, we select seven Gold candidates shown in Figure 2. Target IDs 86861, 93564, 90675, and 92616 are all spectroscopically confirmed at $z \gtrsim 3.07$ and are coincident with compact F160W regions, except for 92616, where there is no F160W imaging. In the latter case, the F336W centroid is coincident with the ground-based Subaru optical broadband counterpart and inside the more extended Ly $\alpha$ emission. IDs 

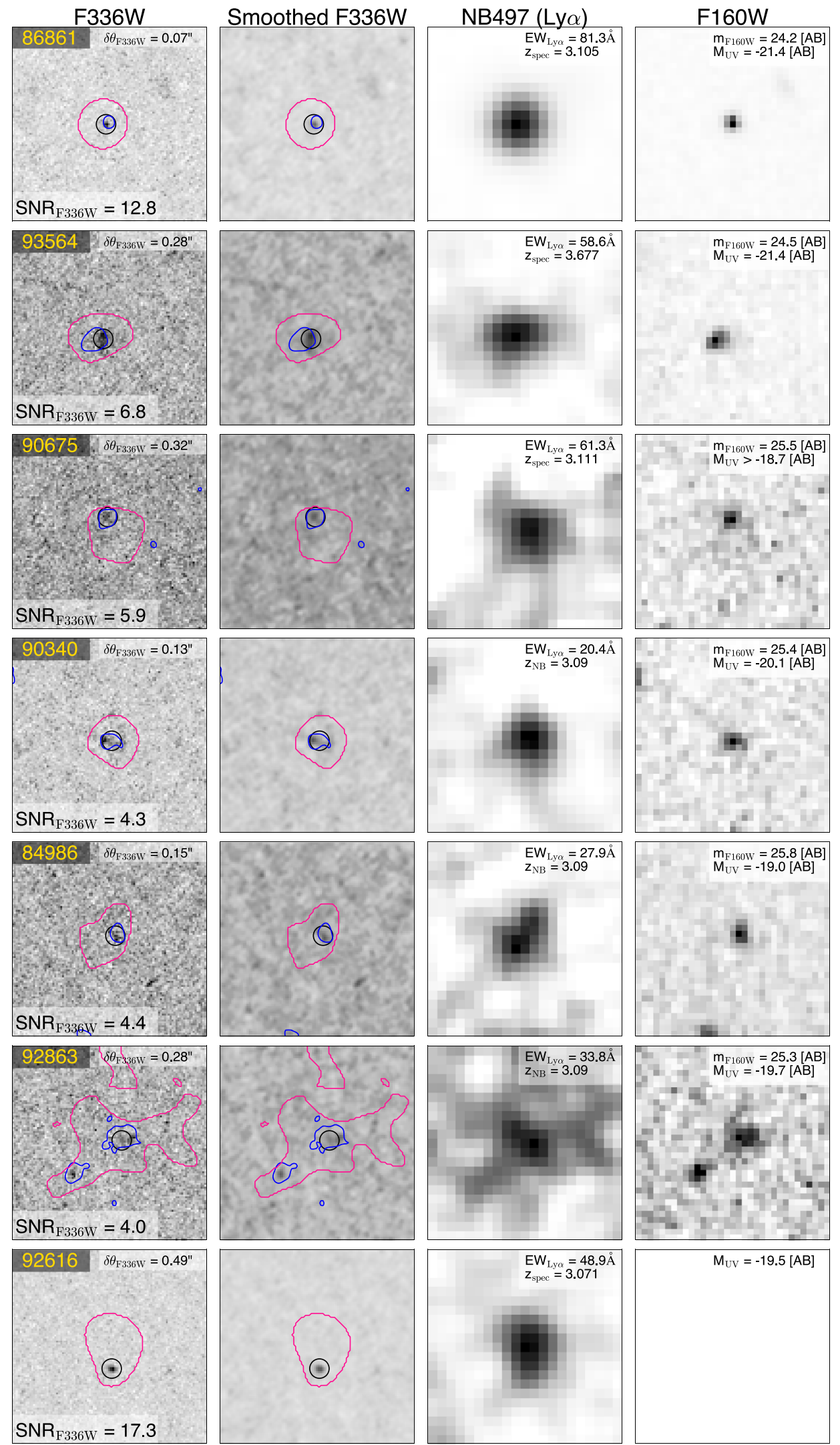

Figure 2. Mosaic of astrometrically aligned $4 \times 4$ !" images for sources with high-S/N F336W detections (see text for discussion of the detection procedure) comprising the Gold subsample. From left to right each panel displays (i) the background-subtracted F336W image overlaid with contours from the narrowband $497 \mathrm{~nm}$ (magenta) and F160W (blue) images and the location of the corresponding F336W source (black circle; 0 !"02 radius), (ii) the former smoothed, (iii) the Subaru narrowband $497 \mathrm{~nm}$ image, and (iv) the F160W image (where available), along with a summary of physical properties where the S/N refers to the F336W detection. All of our objects are LAEs except for 86861, which is an LAE-AGN. 

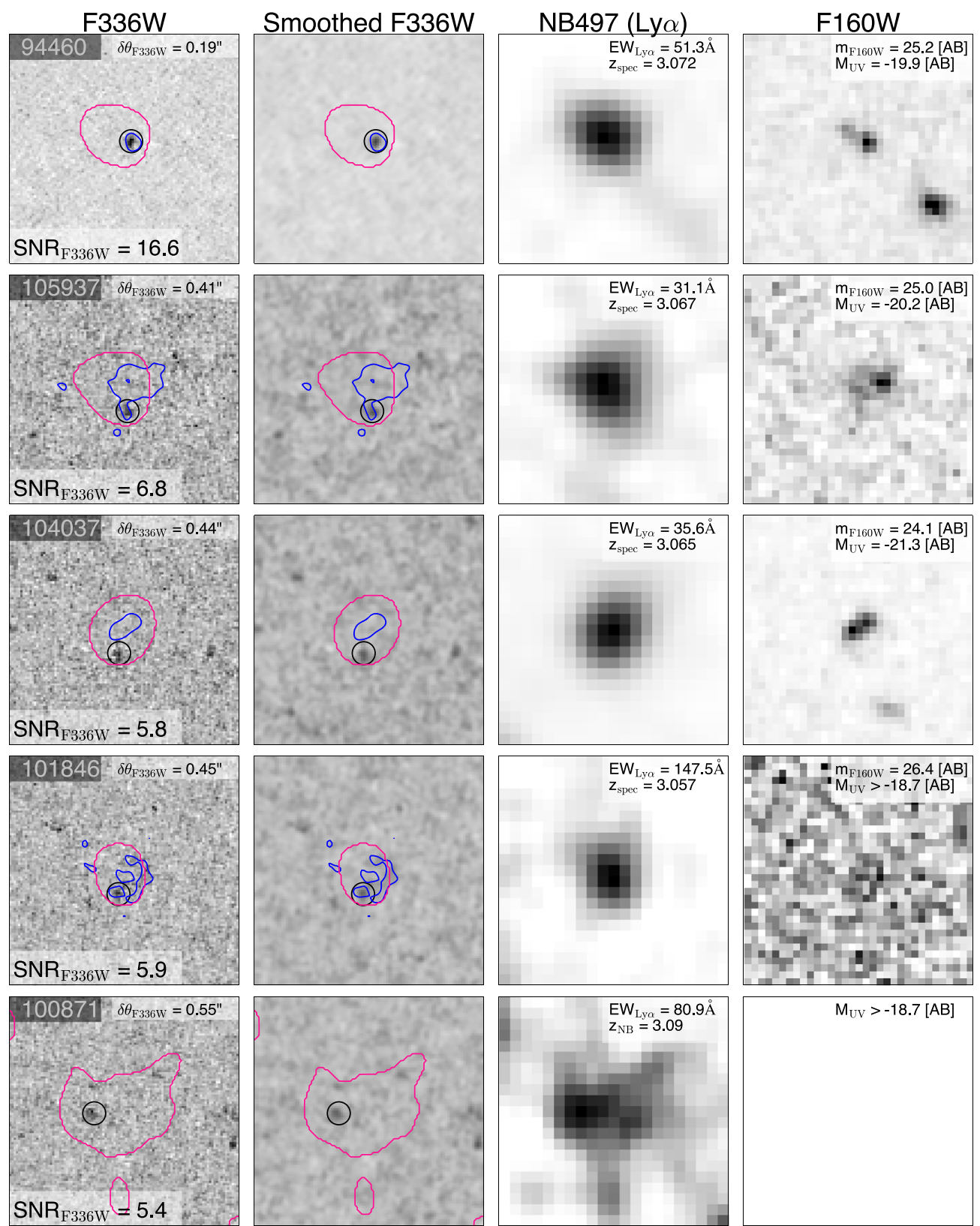

Figure 3. Same as Figure 2, but for the Silver subsample.

84986 and 90340 are considered worthy of inclusion because in both the MOSFIRE spectra no other lines were detected, suggesting that an interloper is unlikely. IDs 92863 and 100871 were not targeted with IR spectroscopy.

We show the Silver subsample in Figure 3. These Silver sources (all $\geqslant 4 \sigma$ ) have more complicated morphologies or less conclusive spectroscopic information but otherwise would qualify for the Gold subsample. ID 94460 is placed in the Silver sample because, in addition to $\mathrm{Ly} \alpha$, [O III], and $\mathrm{H} \beta$ all at $z=3.07$, a spatially offset emission line inconsistent with this redshift was found, which may indicate a contaminating source. IDs 104037 and 105937 fall into the Silver sample owing to their extended F160W regions. Although 100871 also has no F160W imaging and 101846 has only a faint F160W source, their F336W signals are coincident with the peak of Ly $\alpha$ emission.

\subsection{Spatial Offsets}

In considering the validity of our sample, we now examine two further criteria. The first is the distribution of separations between the F336W centroid and that for F160W and Subaru Ly $\alpha$. Although a precise spatial coincidence of LyC leakage and UV/optical continuum and/or Ly $\alpha$ emission is desirable, previous studies have already found that the $\mathrm{LyC}$ emission is occasionally offset from that of Ly $\alpha$ (Iwata et al. 2009; Mostardi et al. 2013, 2015; Micheva et al. 2017b), with a median separation of $\sim 5$ proper kpc reported in Mostardi et al. (2015).

Figures 2 and 3 indicate that, for the majority of our targets, the F336W centroid falls perfectly within the contours from the rest-frame optical (F160W) continuum where available. Figure 4 shows the distribution of spatial offsets between F336W and both the Ly $\alpha$ and F160W centroids. For 75\% of 
Table 4

Properties of the LyC-leaking Candidates

\begin{tabular}{|c|c|c|c|c|c|c|c|c|c|c|}
\hline ID & $\begin{array}{c}f_{\mathrm{Lyc}} \\
\left(10^{-9} \mathrm{Jy}\right)\end{array}$ & $\mathrm{S} / \mathrm{N}$ & $M_{\mathrm{UV}}$ & $\begin{array}{c}\mathrm{EW}(\operatorname{Ly} \alpha) \\
(\AA)\end{array}$ & $z_{\mathrm{sys}}$ & $\begin{array}{c}\Delta v_{\text {Ly } \alpha} \\
\left(\mathrm{km} \mathrm{s}^{-1}\right)\end{array}$ & $\begin{array}{c}\mathrm{EW}([\mathrm{O} \text { III] }) \\
(\AA)\end{array}$ & {$[\mathrm{O} \mathrm{III}] / \mathrm{H} \beta$} & $\mathrm{R} 23$ & {$[\mathrm{O} \mathrm{III}] /[\mathrm{O} \mathrm{II}]$} \\
\hline \multicolumn{11}{|c|}{ Gold Sample } \\
\hline 93564 & $6.2 \pm 0.9$ & 6.9 & $-21.35 \pm 0.05$ & $58_{-6}^{+6}$ & 3.6770 & 574.3 & $1040.1 \pm 33.7$ & $8.6 \pm 0.8$ & $9.5 \pm 0.9$ & $10.1 \pm 0.9$ \\
\hline 90675 & $4.6 \pm 0.8$ & 5.3 & $>-18.72$ & $>61$ & 3.1110 & -3.6 & $<66.3$ & $<0.4$ & $\ldots$ & $\ldots$ \\
\hline 90340 & $4.3 \pm 1.0$ & 4.3 & $-20.08 \pm 0.11$ & $20_{-6}^{+7}$ & $\ldots$ & $\ldots$ & $<55.0$ & $\ldots$ & $\ldots$ & $\ldots$ \\
\hline 92616 & $14.5 \pm 0.8$ & 17.3 & $-19.51 \pm 0.19$ & $48_{-10}^{+12}$ & 3.0714 & 253.3 & $\cdots$ & $>6.2$ & $\cdots$ & $\cdots$ \\
\hline \multicolumn{11}{|c|}{ Silver Sample } \\
\hline 94460 & $16.9 \pm 1.0$ & 16.6 & $-19.88 \pm 0.13$ & $51_{-7}^{+8}$ & 3.0723 & 157.5 & $384.9 \pm 22.6$ & $8.4 \pm 1.8$ & $8.4 \pm 1.8$ & $>10.9$ \\
\hline 105937 & $6.2 \pm 0.9$ & 6.8 & $-20.22 \pm 0.09$ & $31_{-6}^{+7}$ & 3.0668 & 143.7 & $103.4 \pm 15.7$ & $2.4 \pm 0.5$ & $\cdots$ & $\cdots$ \\
\hline
\end{tabular}

Note. $S / N \geqslant 4$ is required for a detection. Column (1): LyC flux. Column (2): LyC S/N. Column (3): absolute UV magnitude. Column (4): rest equivalent width (EW) of Ly $\alpha$. For the $z \simeq 3.1$ objects, the EW is estimated from the BV-NB497 color in conjunction with the Ly $\alpha$ redshift. The EW of LAE93564 is derived from spectroscopy. Column (5): systemic redshift measured from the [O III] and $\mathrm{H} \beta$ line(s). Column (6): velocity offset of Ly $\alpha,\left(z_{\text {Ly } \alpha}-z_{\text {sys }}\right) /\left(1+z_{\text {sys }}\right) \times c$. Column (7): rest EW of [O III] $\lambda \lambda 5007,4959$. The associated continuum is estimated from $H S T / F 160 \mathrm{~W}$ photometry, which is translated into the flux density at $5000 \AA$ with the typical SED of $z \sim 3$ LAEs (Ono et al. 2010). Column (8): [O III] $\lambda \lambda 5007,4959 / \mathrm{H} \beta$. Column (9): R23-index. Column (10): [O III] $\lambda \lambda 5007,4959 /[\mathrm{O}$ II] $\lambda 3727$. No reddening correction has been applied to the oxygen and $\mathrm{H} \beta$ values presented here, as the reddening correction is very small (see Table 5). The asterisk denotes the one LAE-AGN in our sample. Objects 86861, 93564, and 94460 are reported as AGN04, LBG01, and LAE06, respectively, in Micheva et al. (2017a, 2017b).

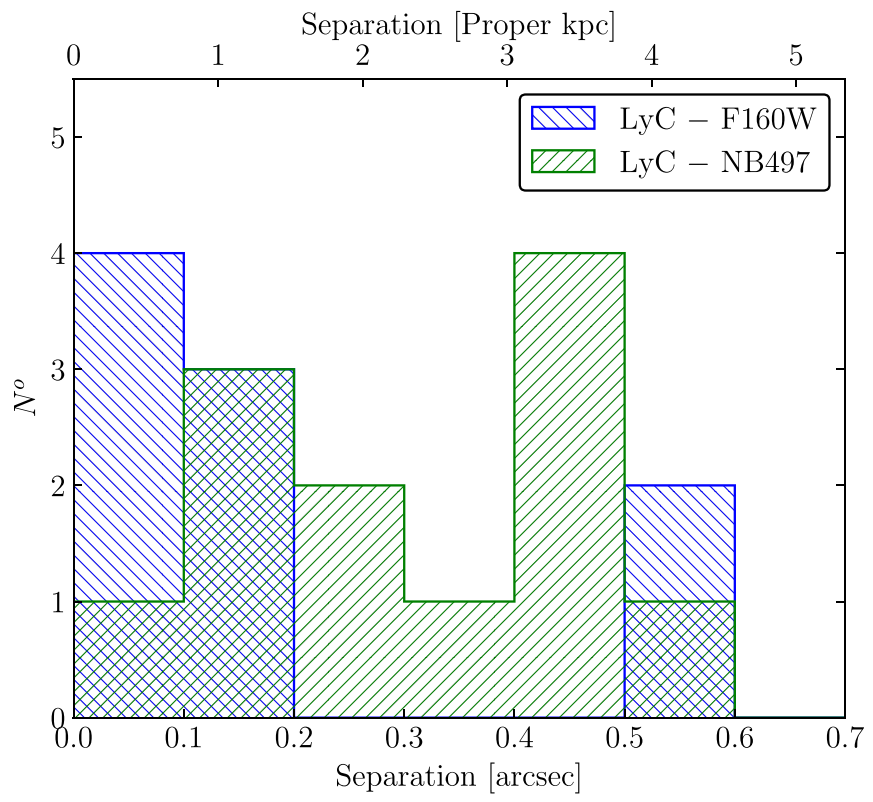

Figure 4. Distribution of separations between the peak of the LyC emission and the peak of both the Ly $\alpha$ (12 cases) and F160W emission (when available, 9 cases).

the candidates, the separation between the LyC and F160W centroid is less than 0 "! 4 ( 3 proper kpc at $z=3.1$ ). For reference $1^{\prime \prime}$ at $z=3.1$ corresponds to 7.6 proper $\mathrm{kpc}$. The angular resolution of the ground-based NB497 images is naturally worse. However, for all of our candidates the F336W emission lies within 0!' 6 (4.6 proper $\mathrm{kpc}$ ) of the Ly $\alpha$ centroid, which we consider satisfactory given that the seeing in the Subaru image is $\simeq 1$ " 0 .
In fact, targets with larger LyC-F160W separations tend to have extended $\mathrm{F} 160 \mathrm{~W}$ or Ly $\alpha$ emission. In these cases the LyC emission is still coincident, but, due to the extended nature of the source, it can fall further from the centroid in the NB497 or F160W bands. If $\mathrm{LyC}$ photons are emitted from regions occupied by young stars, then LyC may reasonably lie closer to the rest-frame optical compared with Ly $\alpha$ that may be resonantly scattered. These small separations are encouraging and lead us to believe that the putative F336W detections are due to LyC photons emitted from LAEs at $z \simeq 3.1$.

\subsection{Foreground Contamination}

Although we have attempted to isolate candidates whose F336W detections may arise from foreground contaminants, we can estimate statistically the likelihood of interlopers from luminosity functions of lower-redshift galaxies. The relevant calculation requires, as input, the aperture within which $\mathrm{LyC}$ flux is searched. As a result, estimates of contamination must account for the possibility that the most active star-forming regions from which LyC photons are emitted could be spatially offset from the bulk of the stars and gas in the galaxy. This effect has been discussed in both ground-based studies (Iwata et al. 2009; Inoue et al. 2011; Nestor et al. 2011, 2013) and those using HST (Mostardi et al. 2015). Allowing for spatial offsets increases the effective aperture and hence increases the likelihood of foreground contamination. In addition, the likelihood of contamination decreases with the depth and resolution of the imaging data. Fortunately, in our case, deep WFC3 UVIS/F336W imaging provides the best angular resolution possible and probes to depths of $30.2 \mathrm{AB}$ mag.

Following the method of Vanzella et al. (2010), we now calculate the probability that a single source is contaminated and the probability that $N$ of our $12 \geqslant 4 \sigma$ detections suffer from 


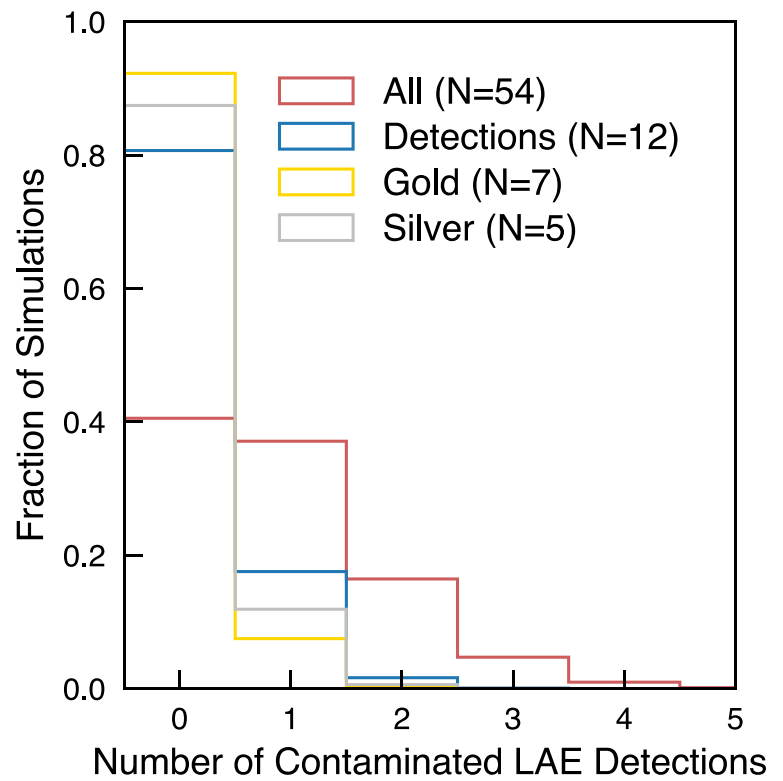

Figure 5. Probability distribution of contamination in our sample of 12 detected objects (blue), and our Gold and Silver subsamples. In $98 \%$ of cases $\leqslant 2$ of the $12 \mathrm{LyC}$ detections are predicted to be affected by contamination. For the Gold subsample, we expect no contamination in $92 \%$ of cases. For the Silver subsample, we expect no contamination in $87.5 \%$ of cases. We also plot the number of contaminates expected in a sample of 54 LAEs, assuming the same LyC offset distribution as our detected sources (red). Even in this much larger sample, $99 \%$ of cases have three or fewer possible contaminates.

foreground contamination. We use the number counts per $\mathrm{deg}^{2}$ in Vanzella et al. (2010) derived from the ultradeep VIMOS $U$-band imaging taken in the GOODS-S field (Nonino et al. 2009). As our F336W measurements are very deep, we use the $3 \sigma$ upper limits for the faintest $U$-band magnitudes extrapolated to $30.5 \mathrm{AB}$ mag (Vanzella et al. 2010). We adopt the distribution of offsets shown in Figure 4, for all our $\geqslant 4 \sigma$ detections, our Gold subsample, and our Silver subsample as apertures for considering possible contamination from the foreground $U$-band sources. In Figure 5 we combine these individual estimates for contamination and run Monte Carlo simulations to show the probability that $N$ of the candidates could be contaminated. The probability that $0,1,2$, or 3 of our 12 candidates could be contaminated is $80 \%, 18 \%, 1.6 \%$, and $<0.01 \%$, respectively. Indeed, in $98 \%$ of cases we estimate that $\leqslant 2$ of our LyC detections could be contaminated. Considering the Gold and Silver samples separately, we expect that these samples would be pure in $92 \%$ and $87.5 \%$ of cases, respectively. We cannot rigorously perform the same analysis on the nondetections, as we cannot measure the possible offsets between LyC and Ly $\alpha$ and F160W centroids. However, if we assumed a similar distribution of offsets, applying the same analysis to all 54 LAEs would still predict far fewer potential contaminants than the number of detections we report for the LACES sample. Quantitatively, we would expect that out of a possible 54 LAEs we would expect $0,1,2,3$, or 4 contaminates in $40.5 \%, 37.1 \%, 16.5 \%, 4.72 \%$, or $0.98 \%$ of cases. We can also use the observed distribution of candidates to estimate the maximum offset of contaminants. One can show that the maximum expected offset of contaminants in the Vanzella et al. (2010) model is related to their mean offset by a geometrical factor of $\sim 1.5$. If we adopt a maximum offset equal to $1.5 \times$ the mean offset of the Gold sample, we would expect $0,1,2,3$, or

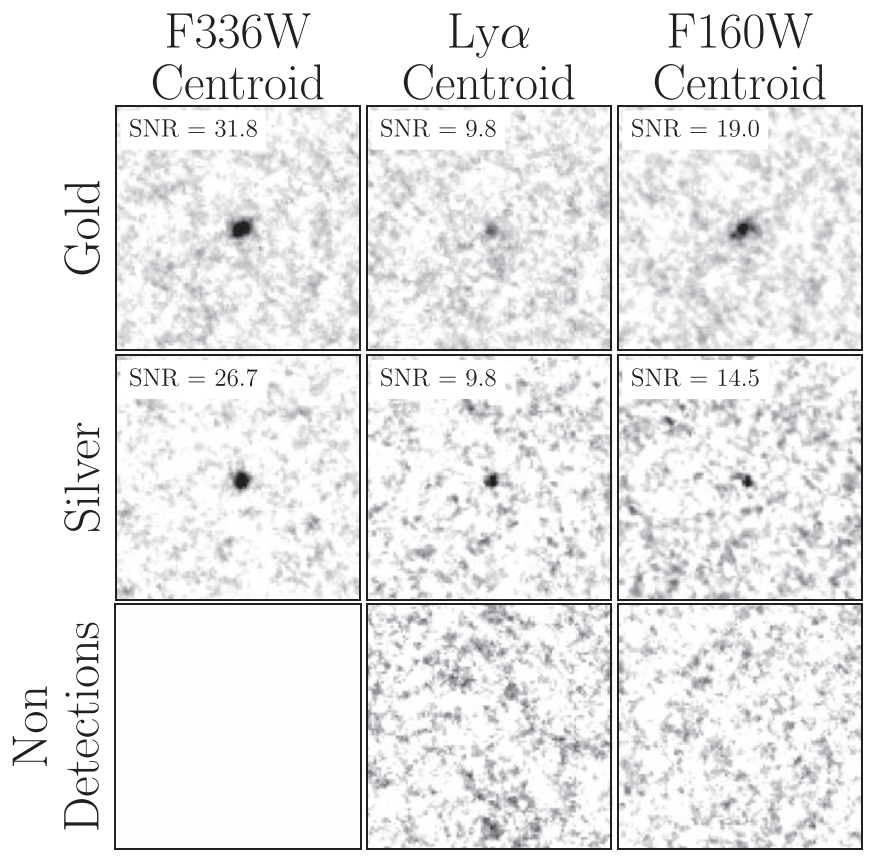

Figure 6. Mosaic of $4 \times 4$ !' images showing stacked F336W images for the Gold (top row), Silver (middle row), and nondetection (bottom row) samples using different methods to center on the candidate galaxies. From left to right each panel displays the detections stacked using (i) F336W centroid, (ii) Ly $\alpha$ centroid, and (iii) F160W centroid or Ly $\alpha$ centroid for cases where F160W is unavailable.

4 contaminates in $33.8 \%, 37.0 \%, 19.9 \%, 6.99 \%$, or $1.8 \%$ of cases assuming 54 possible LAEs. We therefore have identified a robust sample of galaxies displaying Lyman continuum emission.

\subsection{Nondetections}

The majority (42 of 54) of our LAEs have no clear F336W detections above an $\mathrm{S} / \mathrm{N}$ of 4 . To determine whether these nondetections simply represent a tail of fainter signals, we can stack the nondetections to derive a statistical estimate of their mean F336W flux. In this case, we must first consider how to register the images, recognizing that the LyC flux may not always precisely coincide with either the F160W or Ly $\alpha$ signals (Figure 4). We can evaluate the impact of such spatial offsets by conducting the same stacking experiment on those sources for which we see individual detections. By comparing the stack for the Gold and Silver subsamples based on different registrations (F160W and Ly $\alpha$ ), we can compare the loss in stacked signal compared to a direct sum of the registered F336W detections.

For the following stacking procedure, we used custom software based on AstroPy to perform a median stack centered on the position of either the F336W peak (for the Gold and Silver subsamples), the Subaru NB497 Ly $\alpha$ peak, or the HST F160W peak. The F336W frames were smoothed with a 1 pixel rms width Gaussian before stacking to account for small relative offsets or astrometric uncertainties.

The results for the detected (Gold and Silver) subsamples are shown in Figure 6. As expected, using the F160W centroid generally gives a better $\mathrm{S} / \mathrm{N}$ in the final stack, and there is little degradation in signal compared to a direct summation of the F336W signals, especially for the Gold subsample. This correspondence simply reflects the small spatial offsets 
involved. The $\mathrm{S} / \mathrm{Ns}$ of the Gold (Silver) F336W stacks are $\mathrm{S} / \mathrm{N}=31.8$ (26.7) when using the $\mathrm{F} 336 \mathrm{~W}$ centroid, $\mathrm{S} /$ $\mathrm{N}=9.8$ (9.8) when using the $\mathrm{Ly} \alpha$ centroid, and $\mathrm{S} / \mathrm{N}=19.0$ (14.5) when using the F160W centroid. Strong stacked detections remain even without smoothing, with the Gold sample stacks showing $\mathrm{S} / \mathrm{N}=24.0,6.9$, and 13.8 for when centroiding on $\mathrm{F} 336 \mathrm{~W}$, $\mathrm{Ly} \alpha$, and $\mathrm{F} 160 \mathrm{~W}$, respectively, and with the Silver sample stacks showing similar significance.

Applying the same procedure now to $z \simeq 3.1$ LAEs not detected individually in $\mathrm{F} 336 \mathrm{~W}$, we can only register using the Ly $\alpha(N=42)$ and F160W centroids $(N=36)$. Surprisingly, however, no stacked signal is detected regardless of the centroiding or smoothing method. In Section 6.1 we later eliminate the hypothesis that the nondetected sources are drawn from a different population from the 12 detections presented in Figures 2 and 3, since they sample the same range of $M_{\mathrm{UV}}$, $\mathrm{EW}_{\mathrm{Ly} \alpha}, \mathrm{EW}_{[\mathrm{O} \mathrm{III}]}$, and $\Delta v_{\mathrm{Ly} \alpha}$, as shown in Figure 15. Likewise, the issue of spatial offsets (e.g., LyC-F160W) inherent in any sample should not preclude a faint $\mathrm{F} 336 \mathrm{~W}$ detection since the stacked F336W signal is well detected for the Gold and Silver subsamples (Figure 6). The inevitable and remarkable conclusion, therefore, is that the mean F336W signal in the nondetected sample must be uniformly much fainter than for the detected sample. The $3 \sigma$ upper limits for individual objects are typically $30.2 \mathrm{AB}$ mag, and quantitatively we can say that the $3 \sigma$ upper limit we measure for the F336W stack of 42 nondetections is $31.8 \mathrm{AB}$ mag. In addition to median stacks, we have also performed mean stacks of the images, and they also show nondetections to a comparable $3 \sigma$ upper limit. Thus, it appears that the F336W flux in our total sample is either detected individually or not detected at all. We defer discussion of this important result to Section 6.

\subsubsection{Charge Transfer Efficiency and Stacked Nondetections}

An important consideration for interpreting the lack of F336W flux in the stack of nondetections concerns the possible role of degraded charge transfer efficiency (CTE) in artificially suppressing flux from faint objects. If charge was lost in the readout of the data owing to traps in the detector, then one might expect suppression of very faint signals. Indeed, this physical behavior is known to exist for the WFC3/UVIS detector and is actively mitigated by the STScI pipeline, provided that the electron background for the observations is $\gtrsim 12 \mathrm{e}^{-}$per pixel or charge packet. ${ }^{10}$ Our program used postflashing of the detector to reach this background level, which should allow for the CTE corrections in the STScI pipeline to recover efficiently the flux lost during readout. These CTE correction tools are documented on the STScI website. ${ }^{11}$

However, the nominal flux limits reached by the stacks of individually nondetected objects reach to extremely faint limits ( $\sim 32 \mathrm{AB})$. Given the gain of the WFC3/UVIS detector and the exposure time of our individual frames, we estimate that $1 \sigma$ sources in the F336W stack of nondetections would have a charge of only $\sim 9 \mathrm{e}^{-}$in an individual exposure. According to the WFC3/UVIS CTE tools documentation, charge packets of this size in a background of $12 \mathrm{e}^{-}$would lose $\sim 20 \%$ of their charge during readout. To verify this expectation, we used the wfc3uv_cteforward code by Jay Anderson to simulate the CTE effects on charge packets of different amounts $\left(1-1000 \mathrm{e}^{-}\right)$

\footnotetext{
${ }^{10}$ See http://www.stsci.edu/hst/wfc3/ins_performance/CTE/.

11 See http://www.stsci.edu/hst/wfc3/tools/cte_tools/.
}

in differing backgrounds $\left(0-15 \mathrm{e}^{-}\right)$. We distribute fake sources across a model of the WFC3/UVIS detectors and simulate the charge transfer and readout process. We correct the expected flux in each simulated pixel for geometric distortion applying relative exposure maps of the WFC3/UVIS detectors provided by STScI. We use SEP to identify sources in the CTE-affected simulated images using the same effective apertures used in our analysis, and then we compute the fraction of original charge lost during readout. We find that for $9-12 \mathrm{e}^{-}$sources the CTE loss is $16 \%-20 \%$ for $12 \mathrm{e}^{-}$backgrounds. The CTE loss becomes extremely severe for $\lesssim 5 \mathrm{e}^{-}$backgrounds, but such backgrounds are much lower than those in our observations.

For our stack of nondetections, this loss of charge could then reduce a $1.25 \sigma\left(11 \mathrm{e}^{-}\right.$in a single exposure) source below $1 \sigma$ $\left(9 \mathrm{e}^{-}\right.$in a single exposure) flux levels. We verified this in further simulations where we treated the loss of electrons from 9-15 $\mathrm{e}^{-}$sources owing to CTE as a binomial process where the probability of survival for each electron was $80 \%-84 \%$, using $10^{6}$ realizations of their fluxes to determine how many sources could be pushed below $1 \sigma$ in the final stack of nondetections. Importantly, no sources with charge packets $\geqslant 12 \mathrm{e}^{-}$in a single exposure are expected to be suppressed below $1 \sigma$ flux levels in the final stack of nondetections, although individual (uncorrected for CTE) fluxes would be affected at the $20 \%$ level. We therefore conclude that for the backgrounds in our observations CTE should not strongly influence the stringent F336W flux limits from our stack of nondetections.

\section{Analysis}

We now turn to using our F336W detections and upper limits to derive the escape fraction $f_{\text {esc }}$ of ionizing photons, both on a galaxy-by-galaxy basis for our sample and for the population as a whole. We likewise seek to correlate the escape fractions with our infrared spectroscopic measures of [O III] emission, primarily to test the hypothesis that a high escape fraction is connected with the intense [O III] emission that seems commonplace for star-forming sources in the reionization era.

\subsection{Relative Escape Fractions}

Estimating the escape fraction of Lyman continuum photons requires knowledge about the intrinsic source spectrum before attenuation by interstellar dust in the rest-ultraviolet or by the IGM blueward of Ly $\alpha$. We can use SED modeling to constrain the escape fraction while simultaneously fitting for other galaxy parameters on a source-by-source basis, and we perform that analysis below. However, given the additional uncertainties and model dependencies associated with SED fitting, we now consider estimates of the escape fraction derived directly from the source photometry.

The relative escape fraction of Lyman continuum photons, $f_{\text {esc,rel }}(\mathrm{LyC})$, is often defined in terms of the source flux $f_{900}$ at $\lambda_{\text {rest }}=900 \AA$ and the rest-ultraviolet flux $f_{1500}$ at $\lambda_{\text {rest }}=$ $1500 \AA$ as

$$
f_{\text {esc,rel }}(\mathrm{LyC})=\frac{\left(f_{900} / f_{1500}\right)}{\left(L_{900} / L_{1500}\right) t_{\mathrm{IGM}}},
$$

where $\left(L_{900} / L_{1500}\right)$ is the ratio of the intrinsic spectrum at $\lambda_{\text {rest }}=900 \AA$ and $\lambda_{\text {rest }}=1500 \AA$ and $t_{\mathrm{IGM}}$ is the transmission fraction in the Lyman continuum through the IGM (e.g., Steidel et al. 2001; Inoue et al. 2005; Shapley et al. 2006). Clearly both 


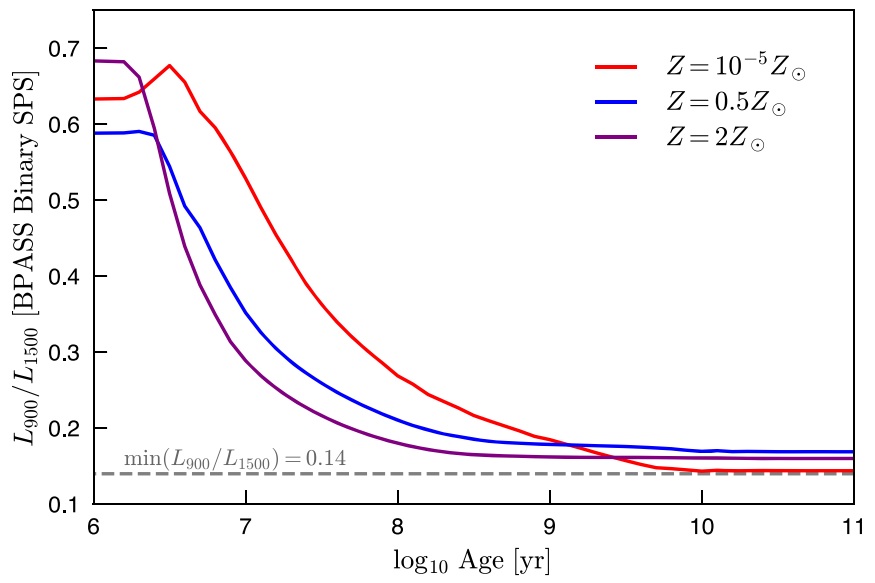

Figure 7. Ratio $L_{900} / L_{1500}$ of the luminosity density at 900 and $1500 \AA$ for constant star formation rate BPASS binary stellar population models as a function of age, for metallicities of $Z=10^{-5} Z_{\odot}$ (red), $Z=0.5 Z_{\odot}$ (blue), and $Z=2 Z_{\odot}$ (purple). For typical ages and metallicities of real galaxies, the luminosity density ratio falls in the range $0.15 \lesssim L_{900} / L_{1500} \lesssim 0.3$. For the estimated relative escape fractions inferred directly from the observed source flux ratios, we will adopt a minimum ratio of $\min \left(L_{900} / L_{1500}\right)=0.14$ (dashed gray line).

$\left(L_{900} / L_{1500}\right)$ and $t_{\mathrm{IGM}}$ are model-dependent quantities and reflect assumptions about the intrinsic stellar population spectrum and the range of IGM absorption properties.

Here we aim to provide an estimate of the relative escape fraction that is easily interpreted before turning to a more sophisticated estimate derived from the full set of photometric data. We therefore define the estimated relative escape fraction as

$$
\tilde{f}_{\mathrm{esc}, \mathrm{rel}}(\mathrm{LyC})=\frac{\left(f_{F 336 W} / f_{R}\right)}{\min \left(L_{900} / L_{1500}\right)\left\langle t_{\mathrm{IGM}}\right\rangle} .
$$

Here we replace the measured flux ratio $\left(f_{900} / f_{1500}\right)$ with our closest photometric flux ratio measure $\left(f_{F 336 W} / f_{R}\right)$ using the F336W and $R$ bands. The quantity $\left\langle t_{\mathrm{IGM}}\right\rangle$ is the mean IGM transmission fraction, and, using the Inoue et al. (2014) IGM absorption model, we find that for our $z \sim 3.1$ emitters $\left\langle t_{\mathrm{IGM}}\right\rangle \approx 0.28$ averaged over the $\mathrm{F} 336 \mathrm{~W}$ band. We then need to define the quantity $\min \left(L_{900} / L_{1500}\right)$, the minimum intrinsic luminosity density ratio expected for the source stellar populations. We choose to use the minimum luminosity density ratio in Equation (2) to provide upper limits on $\tilde{f}_{\text {esc,rel }}$ (LyC) for our choice of $\left\langle t_{\mathrm{IGM}}\right\rangle$. As we will see, the estimated relative escape fraction can be $\tilde{f}_{\text {esc,rel }}(\mathrm{LyC})>1$, which physically requires stellar populations with ages $t<10^{9}$ yr and/or a low-opacity sight line through the IGM.

Figure 7 shows the intrinsic ratio of the Lyman continuum and rest-UV luminosity densities for constant star formation rate binary stellar population models computed using Version 2.1 of the Binary Population and Spectral Synthesis (BPASS) code (Eldridge \& Stanway 2009, 2012; Stanway et al. 2016; Eldridge et al. 2017). We compute the intrinsic ratio as a function of stellar population age in the BPASS models and plot the quantity for metallicities $Z=\left[10^{-5} Z_{\odot}, 0.5 Z_{\odot}, 2 Z_{\odot}\right]$ assuming an upper stellar-mass limit of $M=100 M_{\odot}$. For galaxies with stellar population ages older than $10^{8} \mathrm{yr}$, the typical ratio will be $0.15 \lesssim L_{900} / L_{1500} \lesssim 0.3$. Motivated by the

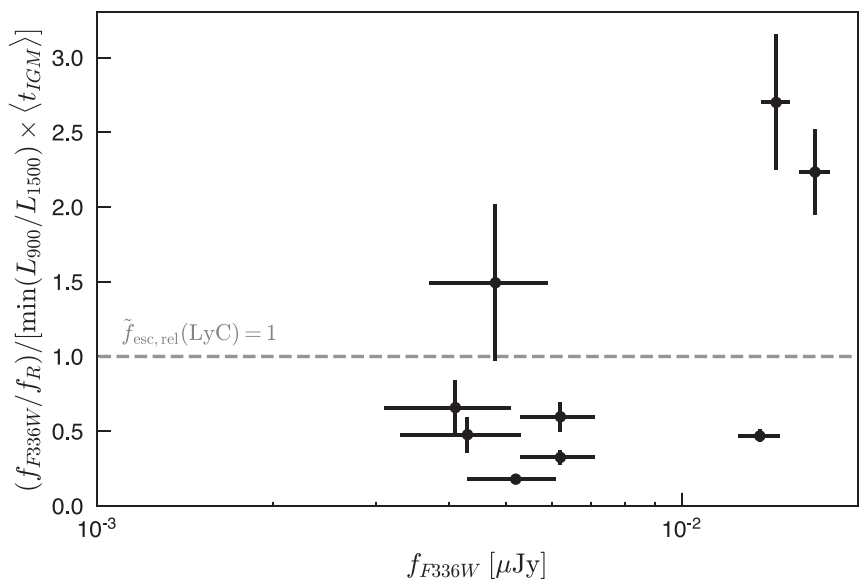

Figure 8. Estimated relative escape fractions $\tilde{f}_{\text {esc, rel }}$ for our LAE sample with $R$-band detections, as a function of the detected F336W flux. Shown are the flux ratios $f_{F 336 \mathrm{~W}} / f_{R}$ (black points) normalized by the minimum intrinsic luminosity density ratio $\min \left(L_{900} / L_{1500}\right)=0.14$ and the mean IGM transmission fraction $\left\langle t_{\mathrm{IGM}}\right\rangle \approx 0.28$. The vertical error bars indicate the uncertainty on the estimated relative escape fractions due to the individual uncertainties on the measured F336W and $R$-band fluxes, while the horizontal error bars correspond to the uncertainties on the F336W flux. For objects above $\tilde{f}_{\text {esc, rel }}=1$ (gray dashed line), young stellar populations $\left(t \lesssim 10^{9} \mathrm{yr}\right.$ ) and/or lower-than-average IGM absorption are required.

behavior of these models, for our estimated relative escape fraction $\tilde{f}_{\text {esc,rel }}$ we adopt the value $\min \left(L_{900} / L_{1500}\right)=0.14$.

With concrete values for the quantities in the numerator of Equation (2), we can use the measured F336W and $R$-band fluxes to estimate $\tilde{f}_{\text {esc,rel }}$. Figure 8 shows the estimated relative escape fraction for our LAE sources as determined by the F336W and $R$-band flux ratios, assuming $\min \left(L_{900} / L_{1500}\right)=$ 0.14 and $\left\langle t_{\mathrm{IGM}}\right\rangle \approx 0.28$. The uncertainties on the estimated relative escape fraction are computed by propagating the uncertainties on the measured fluxes, and we include only sources with $R$-band detections. The $\tilde{f}_{\text {esc,rel }}$ values for individual sources are listed in Table 5 and fall in the range $\tilde{f}_{\mathrm{esc}, \mathrm{rel}} \approx 0.18-2.7$.

\subsection{SED Model Fits}

The absolute escape fraction is conventionally defined as the ratio of those LyC photons emerging compared to those intrinsic to the stellar population. A first necessary step, therefore, is to determine the most likely stellar population and dust extinction for each source from which the intrinsic LyC radiation can be predicted. Fortunately, the SSA22 sample has extensive multiband photometry, and so the SEDs of many galaxies are well constrained and provide the basis for this important step. A second requirement is to correct the detected F336W flux upward to allow for line-of-sight absorption in the IGM. The mean IGM opacity increases as a function of redshift and may vary between sources owing to fluctuations in the number of absorbers along the line of sight.

We begin by fitting the SEDs of all the sources in our sample, regardless of whether they have F336W detections. The SED data comprise $U$-band data from the Canada-FranceHawaii telescope; $B, V, R, i^{\prime}$, and $z^{\prime}$ from Subaru; $J$ and $K$ from UKIRT; and F160W from HST and Channel 3 and 4 coverage from IRAC on board the Spitzer Space Telescope. These 
Table 5

SED Parameter Constraints

\begin{tabular}{|c|c|c|c|c|c|c|}
\hline ID & $A_{\mathrm{SFR}}\left(M_{\odot} / \mathrm{yr}^{-1}\right)$ & $t_{\text {age }}\left(\log _{10} \mathrm{yr}\right)$ & $M_{\star}\left(\log _{10} M_{\odot}\right)$ & $E(B-V)$ & $f_{\mathrm{esc}}$ & $\overline{\tilde{f}_{\text {esc,rel }}}$ \\
\hline \multicolumn{7}{|c|}{ Gold Sample } \\
\hline $86861^{*}$ & $9.06 \pm 0.42$ & $8.49 \pm 0.10$ & $9.44 \pm 0.08$ & $<0.003$ & $0.46 \pm 0.05$ & $0.47 \pm 0.04$ \\
\hline 90675 & $12.53 \pm 6.47$ & $6.34 \pm 0.20$ & $7.38 \pm 0.12$ & $0.03 \pm 0.02$ & $0.39 \pm 0.11$ & $\ldots$ \\
\hline 90340 & $4.29 \pm 0.77$ & $7.90 \pm 0.28$ & $8.52 \pm 0.22$ & $0.03 \pm 0.01$ & $0.30 \pm 0.08$ & $0.48 \pm 0.12$ \\
\hline 84986 & $19.39 \pm 7.83$ & $6.32 \pm 0.22$ & $7.57 \pm 0.10$ & $0.03 \pm 0.01$ & $0.26 \pm 0.05$ & $1.49 \pm 0.52$ \\
\hline \multicolumn{7}{|c|}{ Silver Sample } \\
\hline 94460 & $23.81 \pm 2.00$ & $6.51 \pm 0.03$ & $7.89 \pm 0.01$ & $0.036 \pm 0.003$ & $0.33 \pm 0.02$ & $2.23 \pm 0.29$ \\
\hline 105937 & $4.91 \pm 1.44$ & $8.95 \pm 0.30$ & $9.64 \pm 0.22$ & $0.04 \pm 0.01$ & $0.32 \pm 0.07$ & $0.60 \pm 0.10$ \\
\hline 104037 & $10.94 \pm 1.04$ & $8.22 \pm 0.15$ & $9.26 \pm 0.11$ & $0.011 \pm 0.004$ & $0.13 \pm 0.02$ & $0.18 \pm 0.03$ \\
\hline 101846 & $4.92 \pm 2.73$ & $6.47 \pm 0.24$ & $7.08 \pm 0.13$ & $0.03 \pm 0.04$ & $0.42 \pm 0.09$ & $\ldots$ \\
\hline Silver & $3.10 \pm 0.57$ & $8.97 \pm 0.26$ & $9.46 \pm 0.20$ & $0.01 \pm 0.01$ & $0.51 \pm 0.08$ & $0.73 \pm 0.05$ \\
\hline Nondetections & $1.51 \pm 0.14$ & $8.28 \pm 0.17$ & $8.46 \pm 0.14$ & $0.005 \pm 0.004$ & $<0.005$ & $<0.006$ \\
\hline
\end{tabular}

Note. Ellipsis points denote objects with no $R$-band detection, and correspondingly no measure of $\tilde{f}_{\text {esc,rel }}$.

Table 6

Photometry of the LyC-leaking Candidates

\begin{tabular}{|c|c|c|c|c|c|c|c|c|c|c|c|c|}
\hline ID & $u$ & $B$ & NB497 & $V$ & $R$ & $i^{\prime}$ & $z^{\prime}$ & $J$ & F160W & $K$ & [3.6] & {$[4.5]$} \\
\hline \multicolumn{13}{|c|}{ Gold Sample } \\
\hline $86861^{*}$ & 25.9 & 24.8 & 22.8 & 24.2 & 24.2 & 24.6 & 24.8 & $>23.9$ & 24.2 & $>23.4$ & 23.1 & 23.3 \\
\hline 90675 & $>26.3$ & $>26.8$ & 25.9 & $>26.9$ & $>26.9$ & $>26.6$ & $>25.7$ & $>23.9$ & 25.5 & $>23.4$ & $>22.4$ & $>22.3$ \\
\hline 90340 & 26.3 & 26.7 & 25.7 & 25.9 & 25.5 & 25.3 & 24.9 & $>23.9$ & 25.4 & $>23.4$ & $>22.4$ & $>22.3$ \\
\hline 84986 & $>26.3$ & 26.7 & 26.1 & 26.5 & 26.6 & $>26.6$ & $>25.7$ & $>23.9$ & 25.8 & $>23.4$ & $>24.8$ & $>24.5$ \\
\hline \multicolumn{13}{|c|}{ Silver Sample } \\
\hline 94460 & $>26.3$ & 26.0 & 24.6 & 25.5 & 25.7 & 25.4 & $>25.7$ & $>23.9$ & 25.2 & $>23.4$ & $>24.8$ & $>24.5$ \\
\hline 105937 & 26.1 & 26.1 & 25.1 & 25.5 & 25.3 & 25.2 & 25.3 & $>23.9$ & 25.0 & $>23.4$ & 23.8 & 23.9 \\
\hline 104037 & 25.7 & 24.8 & 23.8 & 24.3 & 24.2 & 24.2 & 24.2 & $>23.9$ & 24.1 & 23.4 & 23.8 & 23.9 \\
\hline 100871 & $>26.3$ & $>26.8$ & 25.6 & $>26.9$ & $>26.9$ & $>26.6$ & $>25.7$ & $>23.9$ & $\cdots$ & $>23.4$ & $>24.8$ & $>24.1$ \\
\hline
\end{tabular}

Note. Magnitude limits correspond to $3 \sigma$ flux limits. Ellipsis points denote objects without F160W coverage.

precursor data sets are summarized in Table 2, and the individual photometry is shown in Table 6.

We use BPASS v2.1 to generate synthetic SEDs that we fit to the data. ${ }^{12}$ Assuming a constant star formation history, $Z=0.1 Z_{\odot}$ metallicities, and stellar masses in the range $M \in[0.1,100] M_{\odot},{ }^{13}$ we couple the BPASS models with the MULTINEST (Feroz \& Hobson 2008; Feroz et al. 2009) nested

\footnotetext{
12 Although one of our Gold subsample objects (86861) is a weak LAE-AGN, from here onward we proceed in using the BPASS models for consistency with the rest of our LAEs.

13 Models with a $300 M_{\odot}$ cutoff increase the ionizing flux by $\sim 5 \%$ (Eldridge et al. 2017) given the same rest-frame UV luminosity. The choice of model will therefore introduce a $\sim 10 \%$ uncertainty in $f_{\text {esc }}$.
}

sampler to perform Bayesian parameter estimation on each galaxy's star formation rate $\left(A_{\mathrm{SFR}} \in[0,100] M_{\odot} \mathrm{yr}^{-1}\right)$, stellar age $\left(t_{\text {age }} \in\left[0, t_{\max }(z)\right]\right)$, dust extinction assuming the Gordon et al. (2003) Small Magellanic Cloud reddening law $(E(B-$ $V) \in[0,1])$, and the escape fraction $\left(f_{\text {esc }} \in[0,1]\right)$. When performing parameter estimation, we use flat priors for all parameters. When fitting the BPASS SEDs to the photometry, we have examined both single and binary star stellar populations and report results for binary population models since these fits produce conservatively lower inferred $f_{\text {esc }}$. Nebular continuum and line emission is included following the precepts of Robertson et al. (2010), with the strength of the nebular emission scaling with the Lyman continuum photon 

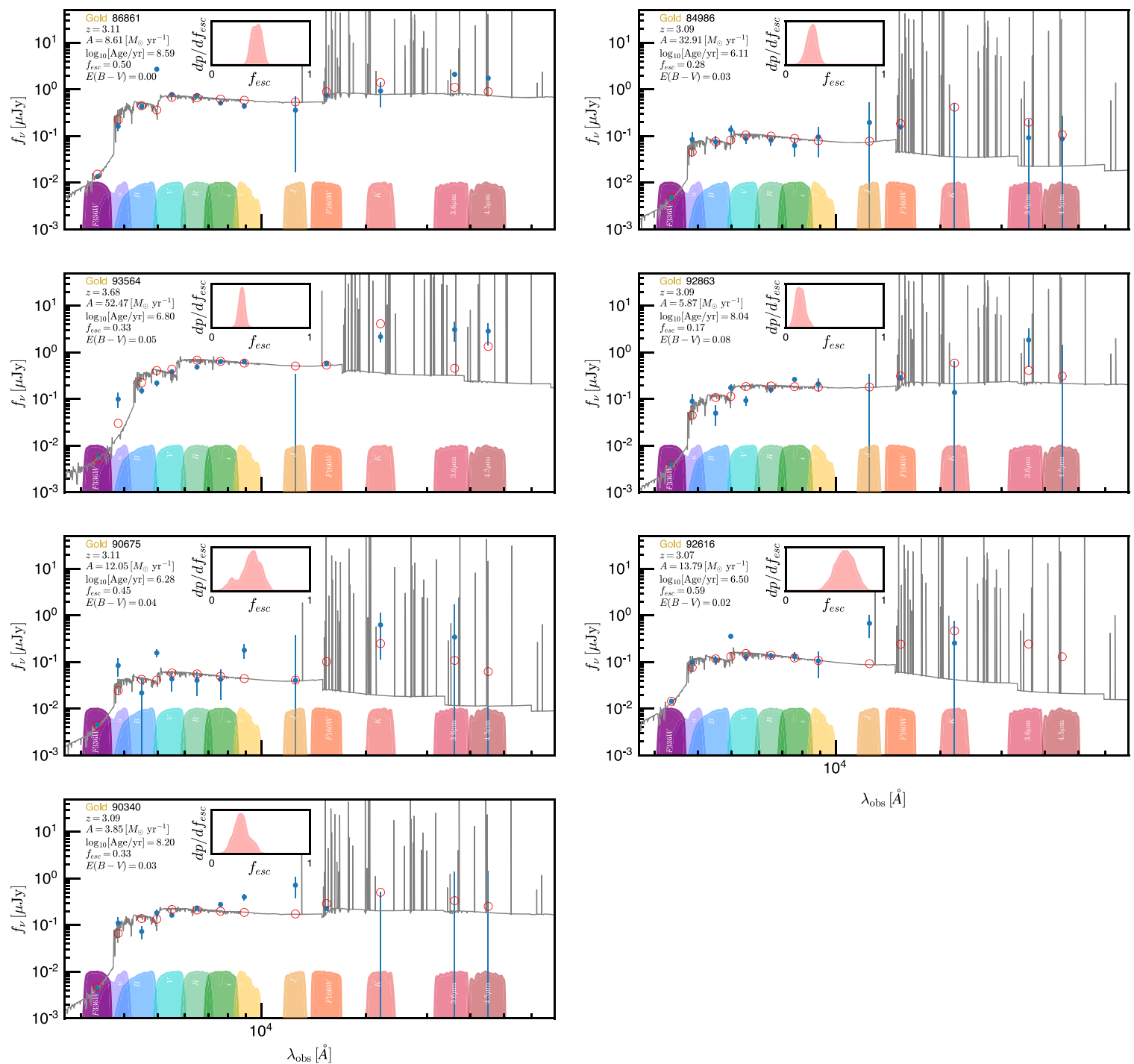

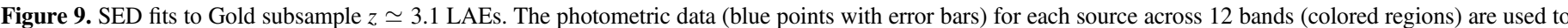

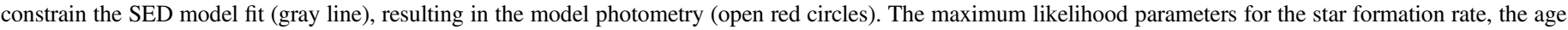

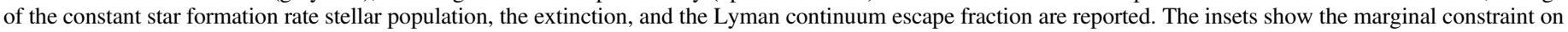
the escape fraction $f_{\text {esc }}$ for each object.

production rate and moderated by the escape fraction. Attenuation from the IGM owing to neutral hydrogen absorption is included following Inoue et al. (2014) and is applied according to the spectroscopic redshift of each source (or the narrowband Ly $\alpha$ redshift if spectroscopy was unavailable). When fit, the escape fraction simply adjusts the model F336W flux by a multiplicative factor, and we incorporate any possible dust attenuation of the Lyman continuum into the value of $f_{\text {esc }}$. However, we do limit $f_{\text {esc }}$ to be less than or equal to the transmission permitted by the dust attenuation expected for a given $E(B-V)$. Model photometry is calculated from the model spectra following Papovich et al. (2001).

Figures 9 and 10 show the model SED fits to the individual Gold and Silver subsample objects. For each object, the maximum likelihood model parameters for star formation rate, stellar age, $E(B-V)$, and escape fraction are indicated. Inset panels in the figures indicate the marginal distribution for $f_{\text {esc }}$ determined from each model fit. The SED parameter constraints are recorded in Table 5, which lists the mean and
$1 \sigma$ width of the posterior distributions. ${ }^{14}$ The quality of the fits vary depending on the photometric constraints available for each object, and the constraints on $f_{\text {esc }}$ vary correspondingly. The typical escape fractions inferred from individual SED fits are $f_{\text {esc }} \approx 0.4$ for the Gold and Silver subsamples, with substantial spread.

\subsubsection{SED Model Tests}

The SED modeling provides stellar population constraints on the objects and enables a model-dependent inference of the escape fraction $f_{\text {esc }}$. While the details of the model do not change whether Lyman continuum flux is detected in our sample and could not permit a conclusion that $f_{\text {esc }} \sim 0$, assessing the influence of our model assumptions on our derived parameters is important. We consider some important potential issues below.

\footnotetext{
14 The error reported here is inferred from the posterior distribution but does not include systematic effects associated with model uncertainties.
} 

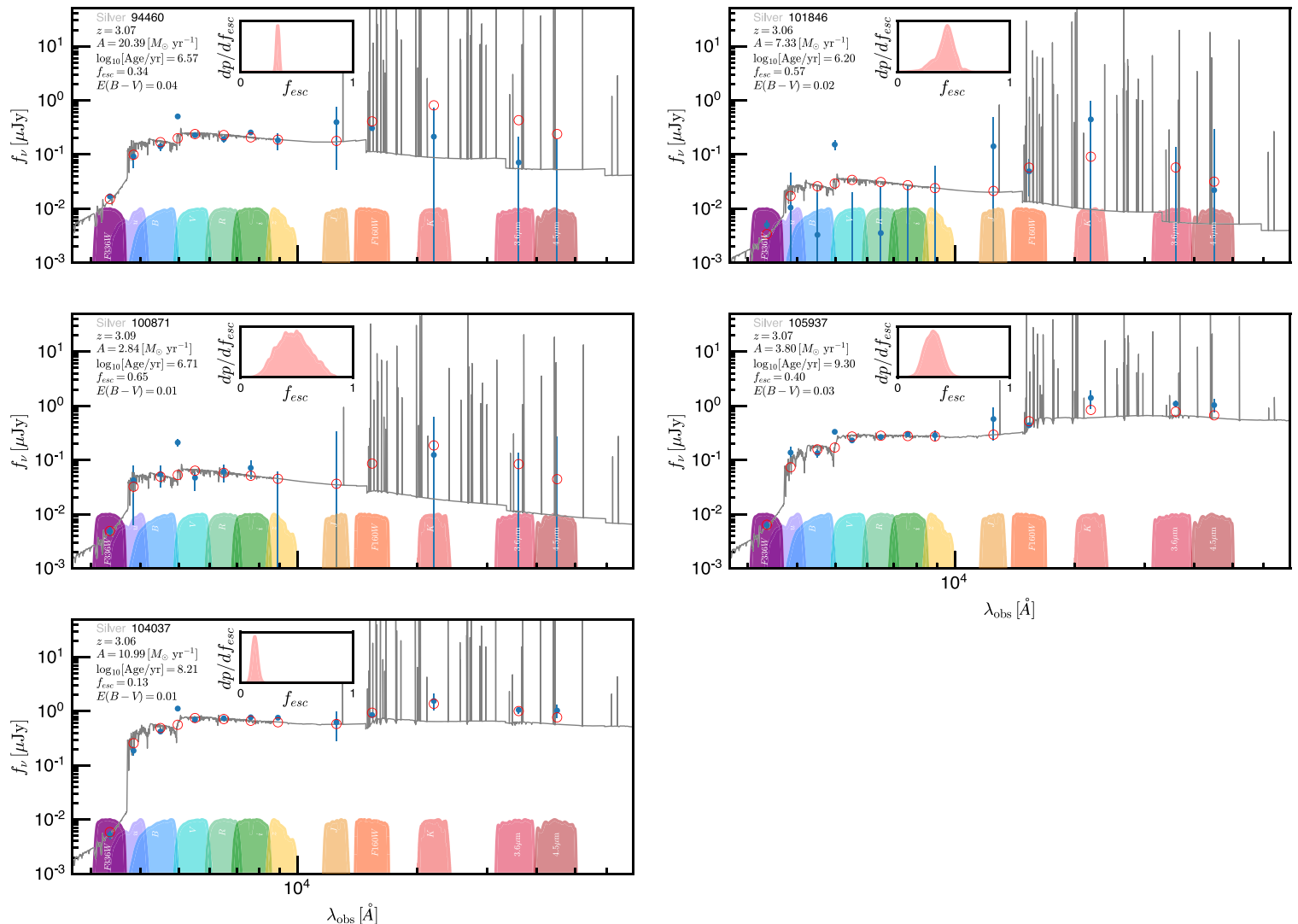

Figure 10. SED fits to Silver subsample $z \simeq 3.1$ LAEs. The photometric data (blue points with error bars) for each source across 12 bands (colored regions) are used to constrain the SED model fit (gray line), resulting in the model photometry (open red circles). The maximum likelihood parameters for the star formation rate, the age of the constant star formation rate stellar population, the extinction, and the Lyman continuum escape fraction are reported. The insets show the marginal constraint on the escape fraction $f_{\text {esc }}$ for each object.

Rest-frame UV and Optical Photometry: The rest-frame optical photometry provides constraints on the presence of evolved stellar populations and, for some combinations of redshifts and photometric bands, the possible influence of nebular line emission. Without constraints on the escaping Lyman continuum flux, the permitted contribution of internally absorbed Lyman continuum photons to the nebular emission can vary widely. Whether the F336W or IRAC fluxes influence the stellar population parameters therefore depends on the detailed shape of the object SED. For instance, Gold Object 86861 has a model escape fraction of $f_{\text {esc }}=0.46 \pm 0.05$ when including all the photometric data. The rest-frame UV, F160W, and IRAC data for this object provide reasonably tight constraints on the object parameters, such that if the F336W data are removed from the fit, the star formation rate and age of the object only change less than $10 \%$ to $A_{\mathrm{SFR}}=$ $8.3 M_{\odot} \mathrm{yr}^{-1}$ and $\log _{10} t_{\mathrm{age}}=8.9$. However, the IRAC data for this object do help resolve the age-SFR degeneracy, and removing the IRAC data decreases the age to $\log _{10} t_{\text {age }}=8.3$, increases the star formation rate to $A_{\mathrm{SFR}}=9.6 M_{\odot} \mathrm{yr}^{-1}$, and decreases the inferred escape fraction to $f_{\text {esc }}=0.38$. For other objects with good photometric constraints in the rest-frame UV and at F160W, such as Silver Object 104037, the escape fraction constraints can change by less than $20 \%$ when the IRAC photometry is ignored.

IGM Absorption: Without considering additional possible constraints on the ionizing emissivity of the LAEs, the escape fraction inferred by the SED modeling will directly anticorrelate with the IGM attenuation along the line of sight to any object. Models of the IGM absorption by Madau (1995) or
Inoue et al. (2014) connect the IGM absorption with the occurrence of neutral hydrogen systems along the line of sight and variations in the absorption to the statistical variance of these absorbers (e.g., Inoue \& Iwata 2008). Since the escape fraction is bounded in the range $f_{\text {esc }} \in[0,1]$ and the IGM absorption depends exponentially on the line-of-sight opacity, the detection of the Lyman continuum in multiple objects may suggest that our SSA22 line of sight has lower-than-average opacity. If the IGM transmissivity is higher than the average $\left\langle t_{\mathrm{IGM}}\right\rangle \approx 0.3$ we assume, then our inferred escape fractions could go down by a factor of two at most. Given that we find a substantial spread in the inferred $f_{\text {esc }}$ for our objects, we can only conclude that the IGM transmissivity is not uniformly low.

Stellar Population Binarity: We assume binary stellar populations in the BPASS models. For a constant star formation rate population with an age $t_{\text {age }}>100 \mathrm{Myr}$, the difference in the Lyman continuum flux per unit UV luminosity density is only $\Delta \log _{10} \xi_{\text {ion }, 0} \sim 0.05$ for $Z=Z_{\odot} / 10$, and the difference in overall production rate of Lyman continuum photons is $\Delta \log _{10} N_{\text {ion }} \sim 0.1$. The differences in $f_{\text {esc }}$ inferred from changing between single and binary stellar populations therefore vary less than the typical uncertainties associated with star formation history, dust, and age.

Dust Model: Variations in the dust model can influence the escape fraction inferred from SED modeling, as the absorption in the rest-frame UV for a given $E(B-V)$ can differ and result in different ratios between the intrinsic model and observed 
Lyman continuum fluxes. Many of our objects are very blue and permit only very small values of $E(B-V)$. For instance, our Gold subsample object 92863 has an inferred SMC $E(B-V)=0.07$. Using a Calzetti et al. (1994) dust law results in an $E(B-V)=0.07$, but the best-fit star formation rate has declined by $40 \%$ to $A_{\mathrm{SFR}}=3.1 M_{\odot} \mathrm{yr}^{-1}$, the age has increased to $\log _{10} t_{\text {age }}=9.3$, and the inferred escape fraction increases substantially to $f_{\text {esc }}=0.39$. However, these changes represent only $\sim 2 \sigma$ changes compared to the SMC dust-based model parameter constraints, and while objects with non-negligible dust may have a systematic uncertainty associated with the dust model, their SED fits tend to be less constrained anyway. Fortunately, our sample of intrinsically blue LAEs will suffer less from the systematic uncertainties associated with dust than Lyman continuum surveys of more evolved LBG samples.

\subsection{Measured versus Model Line Fluxes}

The SED models are fit to the observed photometric fluxes for each source, but our spectroscopic campaign has also provided independent measures of the fluxes of $\mathrm{H} \beta$ and [O III] that can be used to assess the validity of the model line emission that we incorporate in the rest-frame optical source SED. In the case of $\mathrm{H} \beta$, examining the ratio of the model line flux to that observed for a range of the Lyman continuum photon production rate, we find $\left\langle f_{\text {mod }} / f_{\text {obs }}\right\rangle=1.44 \pm 1.81$. Since the model line fluxes depend on $\left(1-f_{\text {esc,SED }}\right)$ determined from the SED fit, this illustrates the degree of selfconsistency between the observed and modeled Lyman continuum flux, the inferred escape fractions $\left(1-f_{\mathrm{esc}, \mathrm{SED}}\right)$, and the method for computing the model line strengths that contribute to the photometric data.

\subsection{Composite SEDs}

The photometry of our sources derives from a combination of ground- and space-based imaging, with a range of sensitivity and spatial resolution. Excepting F336W, the rest-frame UV measurements all come from ground-based data. While these data are of high quality, for some objects it permits a range of stellar population parameters that provide statistically similar model fits. Various combinations of star formation rate and age can produce the same rest-UV flux given these uncertainties but would lead to a range of inferred $f_{\text {esc }}$ as illustrated by the marginal distributions shown in Figures 9 and 10. Given the homogeneity of our sample objects and their comparable redshifts, we have constructed composite photometry for the Gold, Silver, and F336W nondetection subsamples and performed SED model fits to the composite data.

The composite SEDs were generated by first cutting out $6 \times 6$ ". postage stamps, centered on the Subaru positions for each of the LACES LAEs, for all of the available photometric bands. The composites were generated by stacking these cutout images for each band for the Gold, Silver, and nondetected subsamples using the IRAF task imcombine. Only LAEs within the SSA22 protocluster at approximately $z \simeq 3.1$ were included, and LAEs with $z>3.1$, such as ID 93564, were excluded from the composites.

The errors for the composite SEDs were calculated by first masking all objects with $\mathrm{S} / \mathrm{N}>3$ in each image. Regions were then selected from the remaining noise, ensuring no overlap with masked areas of the image. These regions were then used to make stacked images of the noise, stacking the same number
Table 7

Composite SEDs

\begin{tabular}{lccc}
\hline \hline Band & $\begin{array}{c}\text { Gold Sample } \\
\text { Flux (nJy) }\end{array}$ & $\begin{array}{c}\text { Silver Sample } \\
\text { Flux (nJy) }\end{array}$ & $\begin{array}{c}\text { Nondetection Sample } \\
\text { Flux (nJy) }\end{array}$ \\
\hline F336W & $5.29 \pm 0.25$ & $5.86 \pm 0.25$ & $<0.24$ \\
$u$ & $99.7 \pm 15.7$ & $107.7 \pm 17.2$ & $26.2 \pm 7.1$ \\
$B$ & $85.9 \pm 9.2$ & $130.9 \pm 8.5$ & $67.4 \pm 6.0$ \\
NB497 & $226.8 \pm 14.6$ & $370.4 \pm 10.6$ & $259.3 \pm 6.4$ \\
$V$ & $135.2 \pm 10.2$ & $217.8 \pm 8.1$ & $110.3 \pm 6.4$ \\
$R$ & $162.8 \pm 7.8$ & $205.5 \pm 9.6$ & $110.2 \pm 5.3$ \\
$i^{\prime}$ & $194.0 \pm 11.3$ & $257.0 \pm 12.0$ & $112.6 \pm 6.6$ \\
$z^{\prime}$ & $216.9 \pm 20.5$ & $196.6 \pm 23.2$ & $120.1 \pm 11.3$ \\
$J$ & $381.2 \pm 189.4$ & $292.2 \pm 197.8$ & $175.3 \pm 78.9$ \\
F160W & $267.6 \pm 16.3$ & $306.2 \pm 16.0$ & $111.9 \pm 6.9$ \\
$K$ & $248.2 \pm 301.8$ & $809.6 \pm 326.2$ & $123.8 \pm 120.4$ \\
{$[3.6 \mu \mathrm{m}]$} & $654.8 \pm 150.5$ & $591.3 \pm 60.6$ & $340.3 \pm 72.1$ \\
{$[4.5 \mu \mathrm{m}]$} & $764.0 \pm 213.6$ & $462.5 \pm 130.1$ & $286.1 \pm 50.8$ \\
\hline & & &
\end{tabular}

of images used in the Gold, Silver, and nondetection composites $(N=6,5$, and 42 , respectively). This process was repeated across every photometric band for 500 stacks of the noisy regions, and the $1 \sigma$ upper limits were calculated.

Table 7 provides the flux density and associated $1 \sigma$ uncertainty measured in each band for the composite stacks for our Gold, Silver, and nondetection subsamples. Using the higher-precision composite SEDs, we again perform our SED model fits to explore possible inferred differences between the composite properties of our subsamples.

\subsubsection{Gold Subsample Composite SED}

Figure 11 shows the maximum likelihood model fit to the stacked photometry of the Gold sample. This composite at $z \approx 3.1$ appears to be consistent with a very young starforming population, a reddening of $E(B-V) \approx 0.06$, and an escape fraction of $f_{\text {esc }} \approx 0.2$. This SED-inferred escape fraction is lower than the relative escape fraction suggested by the ratio of the 900 and $1500 \AA$ rest-frame flux densities and the typical $f_{\text {esc }}$ suggested by the individual Gold subsample object fits. This discrepancy results from improved constraints on the restframe UV portion of the spectrum gained by stacking. Compared to the other composites discussed below, the mean age is younger although less certain. This reflects the wider range of inferred ages in the individual objects in the Gold sample as listed in Table 5. If the mean age were increased, making it more consistent with that for the other composites, the inferred escape fraction for the Gold sample would be larger. We note that the SED model does not fit the IRAC data particularly well, but for the composite Gold sample photometry ignoring the IRAC data entirely changes the maximum likelihood parameters by $<10 \%$ fractionally. For the escape fraction, fitting the composite Gold sample with or without including the IRAC data changes the maximum likelihood fit by only $\Delta f_{\text {esc }}=0.01$.

\subsubsection{Silver Subsample Composite SED}

Figure 12 shows the maximum likelihood model fit to the stacked photometry of the Silver subsample. Relative to the Gold subsample composite, the Silver subsample composite at $z \approx 3.09$ is consistent with being older and forming stars at a lower rate and with a lower reddening $E(B-V) \approx 0.01$. The inferred Silver composite escape fraction is higher than for the 


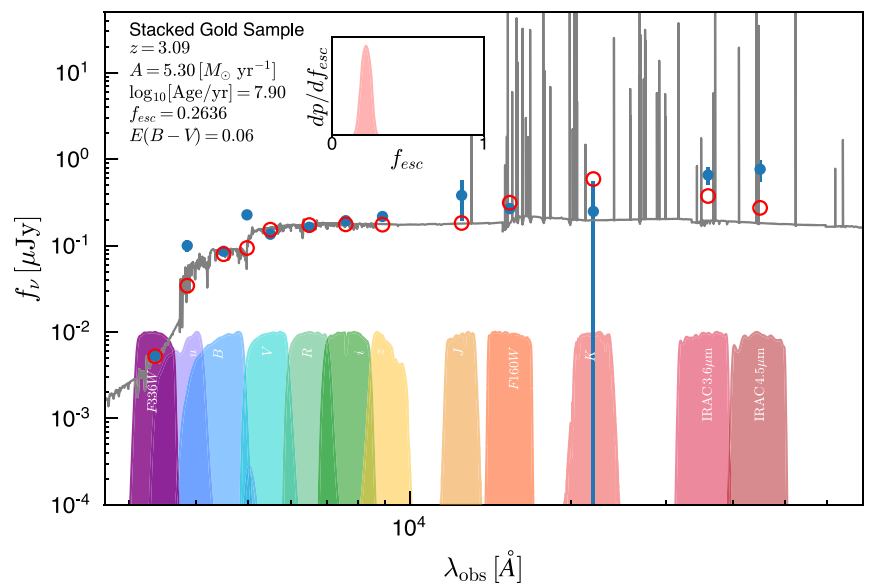

Figure 11. SED model fit to photometry of stacked Gold sample detections.

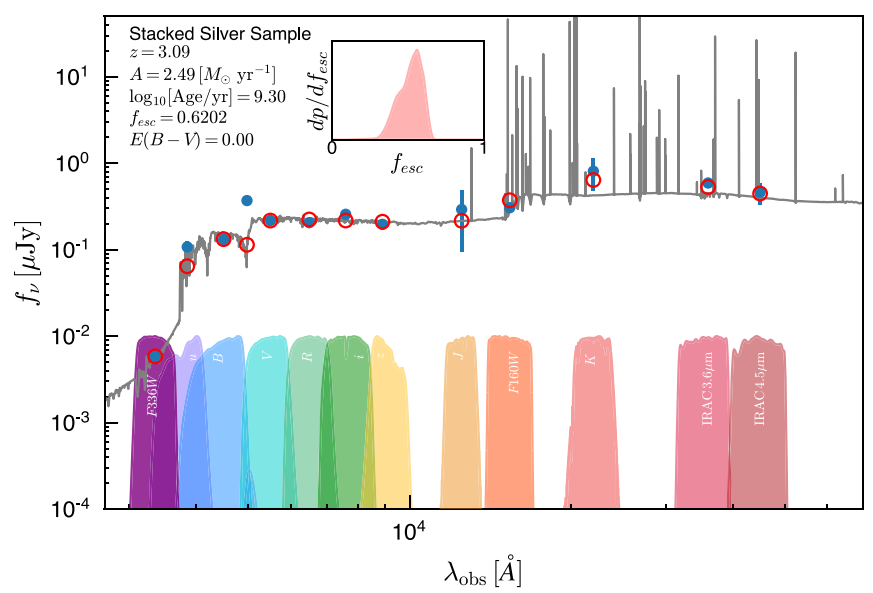

Figure 12. SED model fit to photometry of stacked Silver sample detections.

Gold composite, with $f_{\text {esc }} \approx 0.5$. How representative the escape fraction inferred for this composite Silver SED is for the Silver sample objects individually is questionable, as the photometry for the individual objects varies widely. The stacked Lyman continuum flux is heavily influenced by 94460 , but this object is faint in IRAC and individually has a very young inferred age with moderate escape fraction. The objects 104037 and 105397 contribute greatly to the rest-frame optical emission of the composite Silver SED, but these objects are fairly bright and one is inferred to be old. The combination of these SEDs leads to an old composite age with a necessarily large escape fraction. Clearly some caution is warranted in generalizing the results of the composite Silver SED fit to all LAEs in our sample.

\subsubsection{F336W Nondetection Subsample Composite SED}

Figure 13 shows the maximum likelihood model fit to the stacked photometry of the F336W nondetection subsample. This composite at $z \sim 3.1$ is consistent with a 500 Myr old stellar population forming stars at $A_{\mathrm{SFR}} \lesssim 2 M_{\odot} \mathrm{yr}^{-1}$, lightly reddened, and with a Lyman escape fraction close to zero (i.e., $\left.f_{\text {esc }}<0.005\right)$. In our models, the low escape fraction results in strong nebular continuum and line emission in the rest-frame optical, and the model photometry agrees well with the stacked photometric data at these wavelengths.

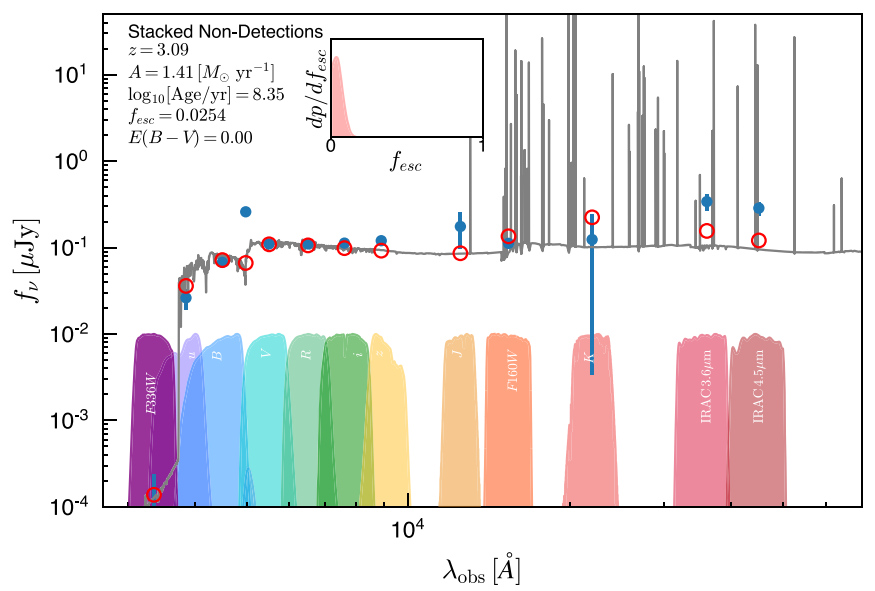

Figure 13. SED model fit to photometry of stacked nondetections.

\section{Results}

We can now take full advantage of our large sample with individually determined $f_{\text {esc }}$ values and investigate possible trends with other galaxy properties so we may better understand the mechanisms through which LyC photons escape.

\subsection{Dependence on the Strength of Lya}

The mechanisms through which $\mathrm{LyC}$ and $\mathrm{Ly} \alpha$ photons escape may be very similar if geometry plays a dominant role in the escape of ionizing photons. Low-density channels created by a burst of star formation may enable LyC and Ly $\alpha$ photons to leak out in a specific direction. However, the radiative transfers of $\mathrm{LyC}$ and $\mathrm{Ly} \alpha$ differ, which may influence their relative visibility. Ly $\alpha$ is absorbed by dust and is a resonant line and can thus be scattered back into the line of sight by neutral hydrogen, whereas LyC photons can only be absorbed by dust and neutral hydrogen. Therefore, it may be possible to have significant Ly $\alpha$ escape while that of Lyman continuum photons is suppressed.

Contrary to this picture, extreme Ly $\alpha$ emission would imply that most of the ionizing photons have been reprocessed as $\mathrm{Ly} \alpha$ photons, allowing few LyC photons to escape. However, Nakajima \& Ouchi (2014) predict that significant $f_{\text {esc }}$ and large $\mathrm{EW}_{\mathrm{Ly} \alpha}$ are possible for $f_{\mathrm{esc}}<0.8$, and it is only for very extreme escape fractions that Ly $\alpha$ will be suppressed.

Many authors have reported a positive correlation between the $\mathrm{EW}_{\mathrm{Ly} \alpha}$ and escaping LyC photons. Verhamme et al. (2017) reported this empirical relation using the small number of confirmed LyC-leaking low-redshift sources. At intermediate redshifts the trend has also been observed using stacked spectroscopy (Marchi et al. 2018) and ground-based imaging (Micheva et al. 2017b), the latter of which may be affected by foreground contamination.

For the LACES sample deep imaging in the Subaru narrowband covering all our targets allows us to determine accurate estimates for the $\mathrm{EW}_{\mathrm{Ly} \alpha}$. We use the Subaru photometry instead of our spectroscopic data because the extended Ly $\alpha$ flux can be included without slit losses and we can easily compare the flux measured in the narrowband to the continuum measured in the broad bands. We show our individually determined escape fractions against $\mathrm{EW}_{\mathrm{Ly} \alpha}$ in the top left panel of Figure 14. There does appear to be a positive correlation albeit with large scatter. Uncertainty in the 

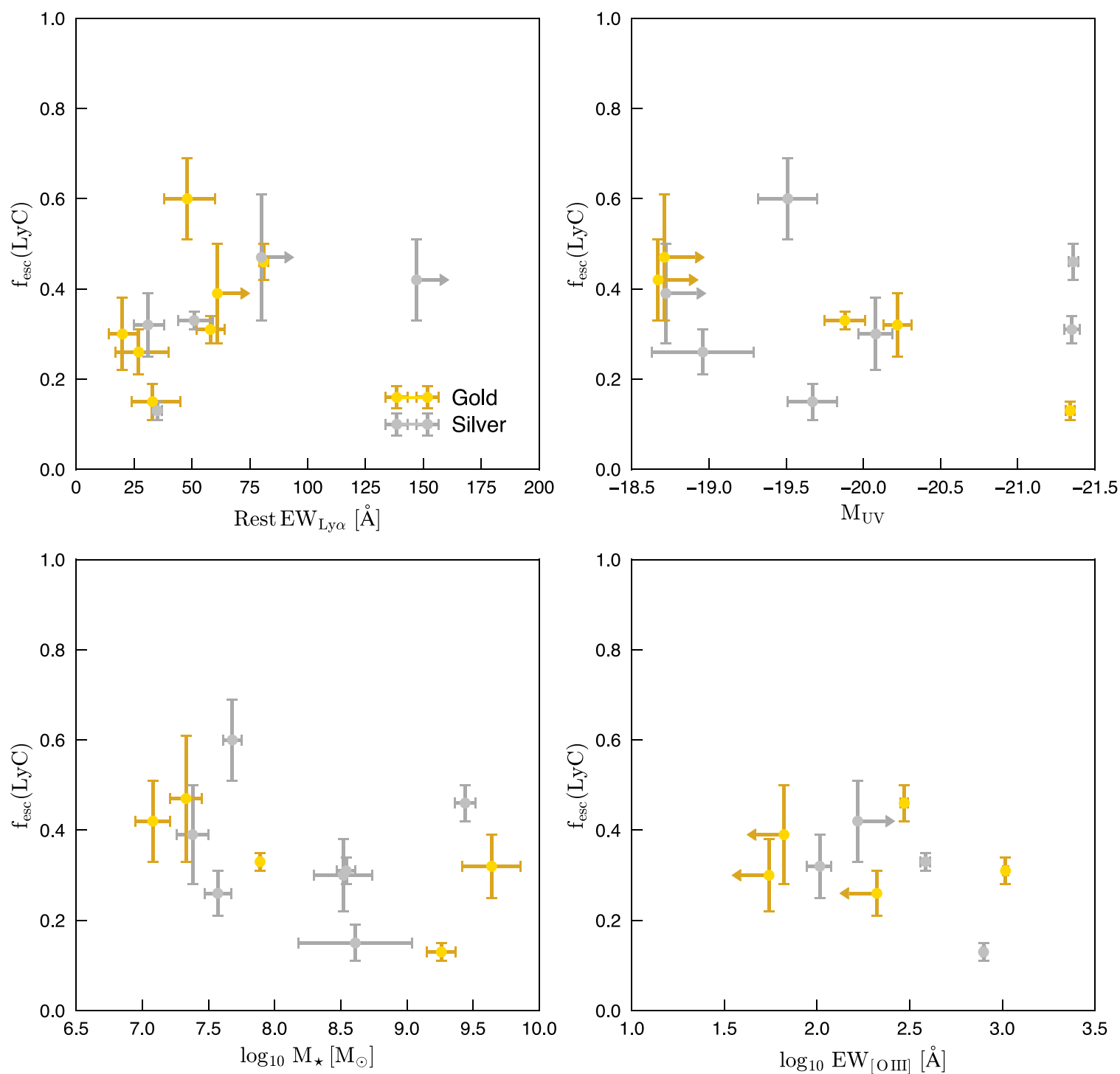

Figure 14. Correlations between $f_{\mathrm{esc}}$ and other observed and derived properties for the LACES Gold and Silver samples. Top left: dependence of $f_{\mathrm{esc}}$ on $\mathrm{EW}_{\mathrm{Ly} \alpha}$. Top right: dependence of $f_{\text {esc }}$ on UV luminosity. Bottom left: dependence of $f_{\text {esc }}$ on stellar mass. Bottom right: dependence of $f_{\text {esc }}$ on the equivalent width of [O III]. In the bottom left panel both $f_{\text {esc }}$ and stellar mass are inferred model-dependent parameters where the stellar mass is the product of the age and SFR derived from SED fitting. Indeed, at fixed age it is expected that the SFR and $f_{\text {esc }}$ would covary to match the F336W measurements and could produce this trend.

likelihood of $f_{\text {esc }}$ arises owing to degeneracies in choosing slightly different models to fit the SED of each galaxy. It should be noted that previous work exploring this correlation at intermediate redshifts did not include such model dependencies, as the observed flux density ratio of LyC to rest-frame UV photons was used instead of $f_{\text {esc }}$.

We also calculate the escape fraction of $\operatorname{Ly} \alpha\left(f_{\mathrm{esc}}^{\mathrm{Ly} \alpha}\right)$ by taking the ratio of the observed Ly $\alpha$ flux to the predicted intrinsic $\mathrm{Ly} \alpha$ flux derived from the observed $\mathrm{H} \beta$ flux using recombination physics,

$$
f_{\mathrm{esc}}^{\mathrm{Ly} \alpha}=\frac{F(\mathrm{Ly} \alpha)}{8.7 \times 2.86 \times F(\mathrm{H} \beta)},
$$

where we use the ratios $\operatorname{Ly} \alpha / \mathrm{H} \alpha=8.7$ and $\mathrm{H} \alpha / \mathrm{H} \beta=2.86$ (Hayes 2015).

A positive correlation between $f_{\mathrm{esc}}^{\mathrm{LyC}}$ and $f_{\mathrm{esc}}^{\mathrm{Ly} \alpha}$ has been reported for the small number of low-redshift LyC-leaking galaxies (Verhamme et al. 2017). The fact that Ly $\alpha$ appears to escape preferentially to LyC may imply that LyC escapes through channels in a "riddled ionization-bounded nebula" (Zackrisson et al. 2013; Behrens et al. 2014; Verhamme et al. 2015), whereas Ly $\alpha$ can escape additionally as a result of resonant scattering. Radiative transfer simulations (Dijkstra et al. 2016) reproduce these trends albeit with much scatter for $f_{\text {esc }}^{\text {Ly } \alpha}>0.1$ owing to the effects of dust, outflow kinematics, and covering factor. For the present sample, no clear correlation between $f_{\mathrm{esc}}^{\mathrm{LyC}}$ and $f_{\mathrm{esc}}^{\mathrm{Ly} \alpha}$ is seen, but uncertainties arising from our faint $\mathrm{H} \beta$ detections may mask a genuine trend.

At first sight, it is puzzling to find a relatively strong correlation between $f_{\mathrm{esc}}^{\mathrm{LyC}}$ and $\mathrm{EW}_{\mathrm{Ly} \alpha}$ but not between $f_{\mathrm{esc}}^{\mathrm{LyC}}$ and $f_{\mathrm{esc}}^{\mathrm{Ly} \alpha}$ when $\mathrm{EW}_{\mathrm{Ly} \alpha} \propto f_{\mathrm{esc}}^{\mathrm{Ly} \alpha} \times\left(1-f_{\mathrm{esc}}^{\mathrm{LyC}}\right) \times \xi_{\text {ion }}$. However, the $f_{\mathrm{eyc}}^{\mathrm{LyC}}-\mathrm{EW}_{\mathrm{Ly} \alpha}$ correlation may have a marginal dependence on $M_{\mathrm{UV}}$. UV-fainter LACES objects tend to have larger $\mathrm{EW}_{\mathrm{Ly} \alpha}$, as seen for many samples in the literature (Stark et al. 2010; Ono et al. 2012; Schenker et al. 2012). This may be due in part to increased $\xi_{\text {ion }}$ (Nakajima et al. 2018). In Section 5.2 we show that $f_{\mathrm{esc}}^{\mathrm{LyC}}$ is anticorrelated with UV luminosity and stellar mass. Therefore, it is not surprising that LAEs with the most extreme 
$f_{\text {esc }}$ and $\mathrm{EW}_{\mathrm{Ly} \alpha}$ are intrinsically faint in $M_{\mathrm{UV}}$. Thus, $f_{\mathrm{esc}}$ correlates with $\mathrm{EW}_{\mathrm{Ly} \alpha}$ because the fainter objects are more compact with a harder $\xi_{\text {ion }}$, which boosts $\mathrm{EW}_{\mathrm{Ly} \alpha}$.

\subsection{Dependence of $\mathrm{f}_{\text {esc }}$ on Luminosity and Stellar Mass}

Understanding the typical $\xi_{\text {ion }}, f_{\text {esc }}$, and $M_{\mathrm{UV}}$ of galaxies at $z>6$ is crucial in determining whether galaxies were the primary driver of cosmic reionization. Current estimates assume an average $f_{\text {esc }}$ and $\xi_{\text {ion }}$ and extrapolate the UV luminosity function down to a limiting magnitude, fainter than current observations (Robertson et al. 2013). It has been suggested that fainter galaxies may have higher $f_{\text {esc }}$ or $\xi_{\text {ion }}$, contributing enough ionizing photons such that galaxies alone are capable of reionizing the universe (e.g., Inoue et al. 2006; Bouwens et al. 2012; Finkelstein et al. 2012; Fontanot et al. 2012, 2014; Kuhlen \& Faucher-Giguère 2012; Robertson et al. 2013; Faisst 2016).

In Nakajima et al. (2018) we have shown for a similar sample of LAEs in the SSA22 protocluster that the production efficiency of ionizing photons, $\xi_{\text {ion }}$, increases toward lower UV luminosities. Grazian et al. (2017) found tentative evidence suggesting a trend between UV luminosity and escape fraction using mostly limits on $f_{\text {esc,rel }}$ derived from ground-based imaging. However, all but three of their points were upper limits for $f_{\text {esc,rel }}$; therefore, this result may simply be due to the $U$-band imaging depth of their observations. It has been suggested that such a correlation could arise from the fact that galaxies with a lower luminosity will tend to also be lower mass and there is an anticorrelation between stellar mass and the $\mathrm{O}_{32}$ ratio (Nakajima \& Ouchi 2014; Dijkstra et al. 2016; Faisst 2016), which itself is expected to correlate with $f_{\text {esc }}$ (Jaskot \& Oey 2013; Nakajima \& Ouchi 2014; Faisst 2016; Nakajima et al. 2016; Izotov et al. 2018). Although, in the bottom left panel of Figure 14, we confirm that the stellar mass is anticorrelated with $f_{\text {esc }}$ for the same objects, this figure is based on two SED-derived parameters, both of which are model dependent.

The top right panel of Figure 14 shows the distribution between $M_{\mathrm{Uv}}$ and $f_{\text {esc }}$ for the individual objects in the LACES Gold and Silver samples. Among these sources, there appears to be no clear correlation between UV luminosities and $f_{\text {esc }}$ despite the correlation between stellar mass and $f_{\text {esc }}$. All the LACES objects presented here are detected LyC leakers. There are a few outliers at the bright end of our sample for which $f_{\text {esc }} \sim 0.5$, although one of these is an LAE-AGN.

\subsection{Dependence of $\mathrm{f}_{\text {esc }}$ on the Strength of [O III]}

There is growing evidence that the $\mathrm{O}_{32}$ ratio correlates with $f_{\text {esc }}$ (Nakajima \& Ouchi 2014; Faisst 2016; Nakajima et al. 2016; Izotov et al. 2018). Characterizing the mean $f_{\text {esc }}$ of $z \sim 3.1$ LAEs with extreme $\mathrm{O}_{32}$ will be useful in understanding the role similar $z>6$ LAEs, where LyC emission is not directly observable owing to a partially neutral IGM, have in contributing to reionization. Unfortunately, due to observational constraints, we did not obtain deep enough [O II] measurements to properly correlate $\mathrm{O}_{32}$ with $f_{\text {esc }}$ for a statistically meaningful sample.

In the absence of [O II] measurements we instead use the EW of [O III], as a large $\mathrm{EW}_{[\mathrm{O} \text { II] }}$ may imply a large $\mathrm{O}_{32}$ ratio. This appears to be a reasonable assumption, and Tang et al. (2018) find that galaxies with large $\mathrm{EW}_{[\mathrm{O} \text { III }]}$ almost always have large
$\mathrm{O}_{32}$. Moreover, it appears that galaxies in the reionization era differ in that they have more extreme [O III], as the strength of the [O III] line appears to increase with redshift (Schenker et al. 2013; Smit et al. 2014, 2015). The discovery that LBGs at $z>7$ with extreme [O III] have been detected with Ly $\alpha$ emission also implies a large $f_{\text {esc }}$, as these objects may have ionized bubbles of hydrogen early, so that their Ly $\alpha$ emission could redshift out of resonance with neutral hydrogen and escape (Oesch et al. 2015; Zitrin et al. 2015; Roberts-Borsani et al. 2016; Laporte et al. 2017; Stark et al. 2017). This assumption appears reasonable, as Tang et al. (2018) find that galaxies with large $\mathrm{EW}_{[\mathrm{O} \text { III] }}$ almost always have large $\mathrm{O} 32$ and large $\xi_{\text {ion }}$.

We use the EW instead of the flux, as our objects span a wide range of magnitudes. Therefore, in order to accurately calculate the $\mathrm{EW}_{[\mathrm{O} \mathrm{III}]}$, we require that our LAEs were targeted and detected in our MOSFIRE campaign but also that our targets were covered by the deep HST F160W photometry, so we can accurately estimate the continuum at the [O III] line (see Section 2.3).

We show the results for the LACES sample in the bottom right panel of Figure 14. There is no clear correlation between $\mathrm{EW}_{[\mathrm{O} \text { III] }}$ and $f_{\text {esc }}$, although the scatter is large. Accordingly, we cannot test the physically motivated hypothesis that densitybounded nebulae result in LyC leakage. Further [O II] measurements would enable us to correlate $f_{\text {esc }}$ directly with $\mathrm{O}_{32}$ for the LACES Gold and Silver subsamples. We will present results from further Keck/MOSFIRE observations of our sample in future work (K. Nakajima et al. 2019, in preparation).

\section{Discussion}

The robust detection of Lyman continuum photons from a substantial subset of our LAE sample, combined with stringent limits on Lyman continuum escape in our nondetected objects, may provide clues as to how galaxies physically release hydrogen-ionizing photons as required if they drove cosmic reionization. Below, we examine possible differences between our detected and nondetected samples, discuss possible physical mechanisms for the escape of Lyman continuum photons that may explain our results, and compare with previous searches for Lyman continuum emission in galaxies.

\subsection{Understanding the Nondetections}

We now discuss the puzzling dichotomy between our LyC detections and nondetections. The 12 LyC-leaking LAEs are all individually detected with $\mathrm{S} / \mathrm{N}>4$ in the F336W images and have $f_{\text {esc }} \sim 15 \%-60 \%$, whereas even in a mean composite spectrum of 38 nondetections we estimate $f_{\text {esc }}<0.5 \%$.

We first investigate whether there are any differences between these two populations in terms of their luminosity $\left(M_{\mathrm{UV}}\right)$, strength of $\mathrm{Ly} \alpha$ emission $\left(\mathrm{EW}_{\mathrm{Ly} \alpha}\right), \mathrm{EW}_{[\mathrm{O} \mathrm{III}]}$, and velocity offset of $\operatorname{Ly} \alpha\left(\Delta v_{\mathrm{Ly} \alpha}\right)$. In Figure 15 we show how the detections, including Gold and Silver subsamples, and the nondetections $(N=42)$ are distributed across these parameters with reference to the full sample of 54 LAEs. It appears that the populations of detections and nondetections are almost indistinguishable from one another. LyC-detected objects span the full range of UV luminosities, as do the nondetections. However, it should be noted that there is an observational bias at fainter luminosities, as we will not be able to detect small 

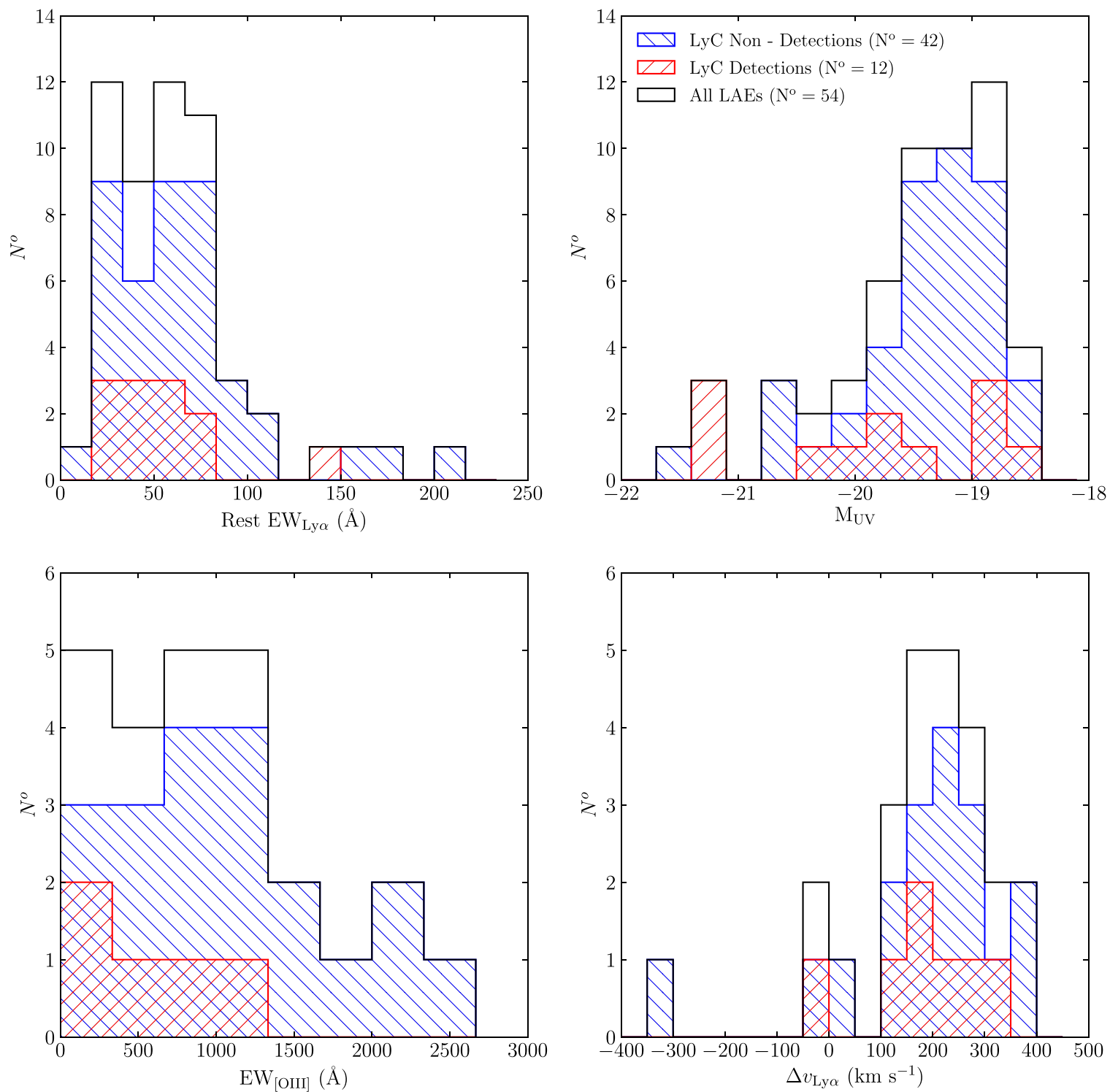

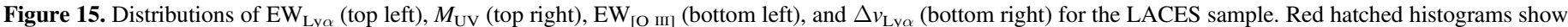

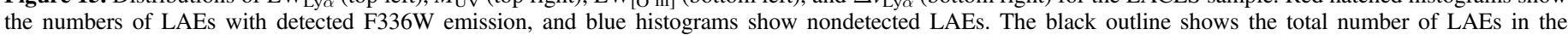

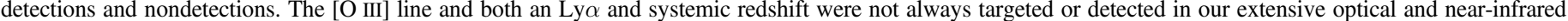

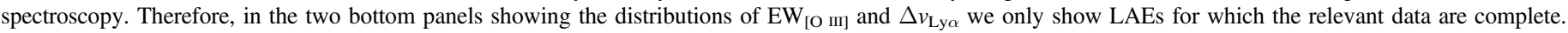

escape fractions in individual cases for the faintest LAEs, and it remains possible that some of the faint LAEs in the LyC nondetections are weak to moderate LyC leakers but below our detection limit. Nevertheless, if this were the case, we would still expect to detect this faint signal in the deep F336W stack, yet we recover on average $f_{\text {esc }}<0.5 \%$.

Additionally, LyC-detected LAEs have a similar distribution in $\mathrm{EW}_{\mathrm{Ly} \alpha}$ with respect to nondetections. However, our LyC detections are almost all at $\mathrm{EW}_{\mathrm{Ly} \alpha}<100 \AA$, as is the case for most of the LAEs in the LACES sample. Of the six LAEs with $\mathrm{EW}_{\mathrm{Ly} \alpha}>100 \AA$, only one is an LyC leaker. This trend may be expected if LAEs with very large $\mathrm{EW}_{\mathrm{Ly} \alpha}$ have reprocessed almost all of their ionizing photons into $\operatorname{Ly} \alpha$, resulting in galaxies with $f_{\text {esc }} \sim 0$ (e.g., Nakajima \& Ouchi 2014). These LAEs are also the faintest objects discussed above, so even if they have a nonzero $f_{\text {esc }}$ it will most likely be below our detection limit. Regardless, the bulk of the nonleaking LAEs contributing to the composite spectrum have moderate $\mathrm{EW}_{\mathrm{Ly} \alpha}$ and $M_{\mathrm{UV}}$ and fall in the same space in an $\mathrm{EW}_{\mathrm{Ly} \alpha}-M_{\mathrm{UV}}$ plot as the bulk of the detections. Therefore, given the evidence from the detections (Sections 5.1 and 5.2), we would have expected these LyC nondetections to have moderate $f_{\text {esc }}$ that would be detectable in our deep F336W stack. Yet, we detect no signal when stacking these objects and find $f_{\text {esc }}<0.5 \%$. This could be due to differences in the covering factor of these LAEs; however, we do not probe this property in our observations.

We also examine the distribution of $\mathrm{EW}_{[\mathrm{O} \text { III] }}$ in Figure 15. Once again, the detections cover the full range of $\mathrm{EW}_{[\mathrm{O} \mathrm{III}]}$, as do the nondetections. If LyC leakage arises as a result of density-bound nebulae with extreme $\mathrm{O}_{32}$ ratios, this may imply extreme $\mathrm{EW}_{[\mathrm{O} \mathrm{III}]}$ (Tang et al. 2018). We may therefore expect the LyC detections to be preferentially clustered at large $\mathrm{EW}_{[\mathrm{O} \text { III] }}$ compared to the nondetections, but the detections fall at $\mathrm{EW}_{[\mathrm{O} \text { III] }} \lesssim 1400 \AA$. Again, it may be the case that if galaxies are leaking a significant fraction of their ionizing photons, there are few $>35 \mathrm{eV}$ photons remaining to doubly ionize oxygen. 
This could perhaps explain why some of our LyC leakers have smaller $\mathrm{EW}_{[\mathrm{O} \text { III] }}$. It is important to note that $\mathrm{EW}_{[\mathrm{O} \text { III] }}$ measurements have not been obtained for all the LAEs, as [O III] was not targeted or detected for every object and we do not have full F160W coverage for the LACES sample in order to estimate the continuum at $\sim 5000 \AA$ in the rest frame.

Finally, we examine the distributions of $\Delta v_{\mathrm{Ly} \alpha}$, which should be $<150 \mathrm{~km} \mathrm{~s}^{-1}$ for LyC leakers that require a low column density of neutral gas for LyC escape (Verhamme et al. 2015). We find no significant difference between detections and nondetections. We will discuss this result in more detail below in Section 6.1.1.

To summarize, $20 \%$ of the LAEs in the LACES sample have individual F336W detections, with inferred escape fractions ranging from $15 \%$ to $60 \%$. Using composite SEDs, we find an average $f_{\text {esc }}=0.20 \% \pm 0.02 \%$ and $f_{\text {esc }}=0.51 \% \pm$ $0.08 \%$ for the Gold and Silver samples, respectively. Even when using a stack of 32 LAEs that are not detected as LyC leakers in individual F336W images, we find on average $f_{\text {esc }}<0.5 \%$ despite these LAEs having an almost identical distribution of luminosities, $\mathrm{EW}_{\mathrm{Ly} \alpha}, \mathrm{EW}_{[\mathrm{O} \text { III] }}$, and $\Delta v_{\mathrm{Ly} \alpha}$ to the LyC leakers. We now turn to possible explanations for this dichotomy.

\subsubsection{Anisotropic LyC Escape}

Previous analyses have proposed (Zackrisson et al. 2013; Behrens et al. 2014; Nakajima \& Ouchi 2014) that there exist at least two mechanisms through which LyC photons can escape. The first involves an ionization-bounded nebula where $\mathrm{H}$ II and $\mathrm{H}$ I shells surround the central stars. LyC photons can escape from such a system if stellar winds or supernovae from a burst of star formation produce low-density holes through the neutral hydrogen in the interstellar medium (ISM). LyC photons can then easily escape through these channels without being absorbed. Alternatively, if the stellar population has a very hard spectrum or there is a significant burst of star formation, the resulting ionization of the gas may enable LyC photons to readily escape in all directions (Zackrisson et al. 2013; Behrens et al. 2014; Nakajima \& Ouchi 2014).

If LAEs are ionization-bound with holes, then it will only be possible to detect LyC leakage if our line of sight is coincident with the opening angle of these channels. All LAEs in a given sample could be leaking LyC radiation, but only a fraction of them, corresponding to the average covering fraction of $\mathrm{H} \mathrm{I}$ and dust, would be detected as LyC leakers through direct observations. The angular dependence of the escape fraction is found to be highly anisotropic in simulations, with galaxies with smaller $f_{\text {esc }}$ having a smaller solid angle through which LyC photons can escape (Paardekooper et al. 2015). Therefore, if the escape of LyC photons occurs anisotropically through channels, it seems likely that our nondetections would have small $f_{\text {esc }}$, with the photons escaping out of small channels directed away from our line of sight. However, we do not probe the covering fraction of our LAEs, and we cannot be certain that geometric effects are the main cause for the dichotomy between our detections and nondetections.

Using Ly $\alpha$ transfer calculations in $\mathrm{H}$ I regions, Verhamme et al. (2015) showed that if LyC escapes owing to an optically thin $\left(N_{\mathrm{H}} \leqslant 10^{18} \mathrm{~cm}^{-2}\right)$, density-bounded regime, then the Ly $\alpha$ profile will be narrow with a small velocity offset $\left(\Delta v_{\text {Ly } \alpha}<150 \mathrm{~km} \mathrm{~s}^{-1}\right)$. However, if the LyC leakers are ionization-bounded and riddled with low-density channels,
$\Delta v_{\mathrm{Ly} \alpha} \sim 0 \mathrm{~km} \mathrm{~s}^{-1}$ with a small red peak due to additional scattered Ly $\alpha$ light that then escapes through the channel. If the dichotomy between our detections and nondetections is caused by geometry, we might expect the LyC leakers to be preferentially clustered around $\Delta v_{\mathrm{Ly} \alpha} \sim 0 \mathrm{~km} \mathrm{~s}^{-1}$ when compared to the nondetections.

In Figure 15 we show the distribution of $\Delta v_{\mathrm{Ly} \alpha}$ for the detections and nondetections where both an Ly $\alpha$ and systemic redshift are available. The LyC detections cover the full range of velocity offsets and are not centered only around small velocity offsets. Therefore, it is not clear that the nondetections are ionization-bounded whereas detections are riddled or density-bounded. Indeed, the Ly $\alpha$ profile is most likely more complex than this simple picture. Velocity offsets could be caused by outflows. Also, if LyC escapes through small offset channels, this geometry could have little implication for the Ly $\alpha$ profile, which may still be dominated by resonant scattering. More detailed analyses of $\operatorname{Ly} \alpha$ spectra at higher spectral resolution are most likely needed before ruling out the geometric picture of Ly $\alpha$ escape.

\subsubsection{Stochastic LyC Escape}

Star formation at these redshifts may be highly time dependent. Galaxies accrete gas from the IGM and through mergers. They undergo bursts of star formation, as a result of which feedback in the form of stellar winds and supernovae can ionize their ISM. During the relatively quiescent periods, the ionized gas will recombine. LyC leakage may therefore be stochastic with bursts of star formation either ionizing all the neutral hydrogen within the virial radius or creating channels through which LyC photons can escape. This has been widely reported in simulations of leaking LyC radiation where $f_{\text {esc }}$ has traced bursty star formation with a time delay of $\sim 10 \mathrm{Myr}$ (Kimm \& Cen 2013; Wise et al. 2014; Ma et al. 2016; Kimm et al. 2017; Trebitsch et al. 2017) and with smaller, lower-mass galaxies expected to be more stochastic.

We would therefore expect that the LACES LAEs with no leaking LyC radiation are being observed in these quiescent periods where the ISM has had time to recombine. However, this picture is difficult to reconcile owing to large $\mathrm{EW}_{[\mathrm{O} \text { III] }}$ we observe for the nondetections, implying very recent star formation. These galaxies may be recently star-forming, but feedback may not have been effective in creating pathways for the radiation to escape. This ineffectiveness may trace an additional factor such as the covering fraction, which could be varying between individual galaxies. Thus, galaxies with lower covering fractions are more able to leak $\mathrm{LyC}$, given the same burst of star formation and feedback.

In this scenario, only a fraction of LAEs at any time would be at a point where they had recently undergone a burst of star formation $\sim 10 \mathrm{Myr}$ ago, and only these LAEs would be detectable as having a nonzero $f_{\text {esc }}$. If it were possible to observe these LAEs over hundreds of megayears, perhaps we would see the LAEs flash "on" and "off" in LyC emission.

\subsubsection{Spatially Varying Intergalactic Medium}

As our study takes place within the SSA22 protocluster, a further possibility is that the IGM is spatially varying on scales of tens to a few hundred megaparsecs within our area of study and the field of view of the F336W images. This density variation would result in LyC leakage from LAEs in regions 


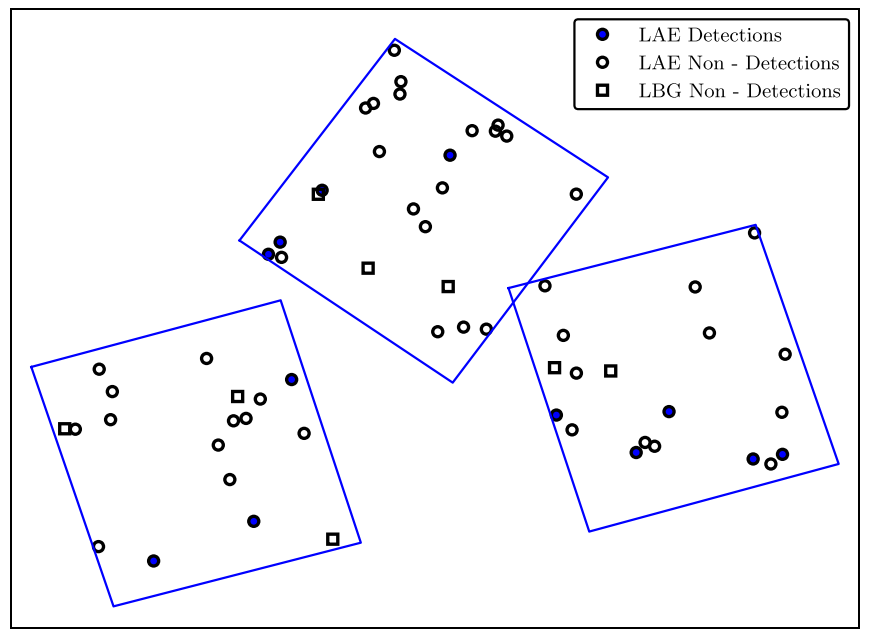

Figure 16. Spatial distribution of LyC detections (blue) and nondetections (white) for all LAEs and LBGs (circles and squares, respectively) in the LACES sample. Detections and nondetections appear to be spatially clustered, which could be due to spatial variations of H I gas in the SSA22 protocluster.

with a higher column density of $\mathrm{HI}$ being more strongly absorbed, and thus we would measure a reduced or zero $f_{\text {esc }}$ for these objects.

Evidence for spatial variation of $\mathrm{HI}$ in the IGM and CGM within the SSA22 protocluster has been investigated in the literature. Using bright galaxies behind the protocluster, Mawatari et al. (2017) measured Ly $\alpha$ absorption in the spectrum of these galaxies due to absorption by $\mathrm{H}$ I within SSA22. They found that SSA22 has an excess of H I compared to similar independent control fields. They also found that there is a large-scale diffuse $\mathrm{H} \mathrm{I}$ component that is independent of the CGM of individual galaxies, that Ly $\alpha$ absorption increased on $<100 \mathrm{Mpc}$ scales possibly owing to the CGM of nearby galaxies, and that stronger LAEs had weaker H I absorption.

Mawatari et al. (2017) focused on the center of SSA22, and the LACES sample is drawn from the edge of the region considered in their study. It is therefore possible that there is large-scale diffuse H I unconnected to individual LAEs within our field and that a particularly dense CGM of a nearby galaxy or an ionizing neighbor with a large $f_{\text {esc }}$ may respectively inhibit or boost the chances of leaking LyC radiation reaching us as observers. Figure 16 shows the spatial distribution of LAE-LyC detections (blue circles) and LyC nondetections for LAEs and LBGs (white circles and squares, respectively).

Indeed, it does seem that the LyC detections and nondetections appear clustered on small scales, which could be due to spatially varying $\mathrm{H}$ I in the IGM and CGM. We cannot directly measure the $\mathrm{HI}$ in the LACES field, but future detections of leaking LyC radiation in clusters with lower $\mathrm{H}$ I density or in blank fields may help us understand whether LyC nondetections in extreme LAEs are due to $\mathrm{H} \mathrm{I}$ absorption. It should be noted that this effect would only change the fraction of leaking LyC radiation that is absorbed along the line of sight. We have still detected LyC escape in a significant fraction of our LAEs, but it is possible that more IGM absorption results in nondetections for the remaining LAEs.

\subsection{Comparison to Other Studies}

Through the LACES program, we have provided a significant number of individual LyC measurements for a homogeneous sample of star-forming galaxies in a narrow redshift interval at $z \simeq 3.1$, corresponding to escape fractions of $f_{\text {esc }} \simeq 15 \%-60 \%$. Of particular significance is the high success rate $(\simeq 20 \%)$ within our sample, one considered to be potential analogs of [O III]strong metal-poor sources in the reionization era.

Early efforts to directly measure significant escape fractions in galaxies at intermediate redshifts have largely been unproductive apart from a few exceptional cases (de Barros et al. 2016; Shapley et al. 2016; Vanzella et al. 2016, 2018; Bian et al. 2017). Without a sizable number of detections drawn from a homogeneous sample, it has therefore been difficult to make progress in understanding under what conditions LyC photons can escape.

An alternative approach when no individual detections can be found, for example, due to the limited sensitivity of the data, is to stack the LyC signal from a large sample either with suitable photometry (Grazian et al. 2017; Japelj et al. 2017; Matthee et al. 2017; Rutkowski et al. 2017; Naidu et al. 2018) or with spectroscopic data (Marchi et al. 2018; Steidel et al. 2018). However, with the exception of the recent campaign by Steidel et al. (2018), this has resulted primarily in upper limits of $f_{\text {esc }}<10 \%$ below the canonical value of $10 \%-20 \%$ required to sustain reionization (Robertson et al. 2013). The results of these programs have led to speculation that reionization may not be driven by star-forming galaxies (e.g., Madau \& Haardt 2015).

An important conclusion from our work, which would not be easily seen in early stacking programs, is the distinction between the 12 LACES LAEs that show convincing individual detections in the range $f_{\text {esc }} \simeq 15 \%-60 \%$ and 42 LAEs that, even when stacked, show no significant leakage consistent with an individual average $f_{\text {esc }}<0.5 \%$. If, as we surmise, LyC leakage is either "on" or "off" owing to anisotropic or timevarying factors, then achieving adequate depth for an individual target is crucial to making the distinction. If $z>7$ sources had the same inferred escape fraction as our LyC detections, they could maintain reionization. Yet shallower surveys of sources less analogous to $z>7$ objects would have concluded the opposite.

As an illustrative example, stacking F275W photometry, Rutkowski et al. (2017) found $1 \sigma$ upper limits of $f_{\text {esc }}<14.0 \%$ for 13 "extreme emission line galaxies" (EELGs) at $z \sim 2.3$ with $\mathrm{O}_{32}>5$. Although $3 \sigma$ upper limits derived from their measurements could still be consistent with significant $f_{\text {esc }}$, this result casts doubt on whether galaxies with extreme $\mathrm{O}_{32}$ are LyC leakers. Similarly, Naidu et al. (2018) find $f_{\text {esc }}<16.7 \%$ for fainter EELGs and $f_{\text {esc }}<8.5 \%$ for their brighter EELGs using stacked ground-based $U$-band imaging. However, if only a fraction of the LyC photons are escaping in our direction, then they will remain undetected in the relatively shallow images used by Rutkowski et al. (2017) ( $3 \sigma$ depth ranging 26.5-28.2 AB mag), whose depth was optimized for a composite stack.

The Steidel et al. (2018) spectroscopic campaign is the only comparative study that reaches a depth adequate for individual LyC detections comparable to escape fractions of $\simeq 10 \%$. Individual detections were seen for $15 / 124$ sources. The approach is highly complementary to the present study in several respects. It focuses on LBGs over a wider redshift range with LyC signals inferred optimally from spectra in a narrow wavelength window (880-910 nm) where IGM absorption is reduced and samples multiple sight lines to reduce cosmic 
variance. The LACES program avoids some of the limitations of earlier HST imaging campaigns that targeted LBGs with a range of redshifts. LACES exploits a narrowband-selected sample of LAEs at $z \simeq 3.1$ optimally matched to the F336W filter and the improved depth of the HST imaging (a $3 \sigma$ limiting magnitude of $\simeq 30.2$ ) to provide exquisite limits on individual sources with the necessary resolution to mitigate issues of foreground contamination.

Comparing the two approaches, the success rate of detecting LyC emission in the LACES sample $(S / N \geqslant 4$ detections for $20 \%$ of the total sample and $21 \%$ for LAEs only) is higher than that seen for individual LBGs $(\simeq 10 \%$ to broadly comparable escape fractions in the Steidel et al. 2018 survey). Indeed, none of the seven LBGs in our LACES control sample have detectable LyC emission. Our earlier work has shown that LAEs have a harder $\xi_{\text {ion }}$ than LBGs (Nakajima et al. 2016, 2018). With more ionizing photons, significant LyC leakage is more likely for LAEs and also results in larger $\mathrm{O}_{32}$ ratios and more extreme [O III] EWs. LAEs can more readily leak LyC photons in riddled ionization-bounded or density-bounded nebulae, physical conditions more easily met for younger, lowmass, and metal-poor galaxies, with important implications for comparable sources in the reionization era.

Despite the different approaches, many of the conclusions of the present paper are supported by the Steidel et al. (2018) results, including the absence of any demographic differences between the sample of individual detections and those nondetected and similar correlations between $f_{\text {esc }}$ and $\mathrm{EW}_{\mathrm{Lya}}$ and $M_{\mathrm{UV}}$. The primary difference remains the higher success rate of detecting LyC leakages in LAEs and the possible association with [O III] emission.

\subsection{Implications for Cosmic Reionization}

Our interest in the LACES sample and this study is motivated, in part, by the likelihood that our $z \simeq 3.1$ LAEs are promising analogs of sources in the reionization era and thus that inferences on the physical conditions that permit LyC photons to escape will have important implications for the assumption that cosmic reionization is largely driven by similar systems.

It is reasonable to assume that LAEs at intermediate redshifts that are metal-poor, low-mass star-forming galaxies are similar to those at higher redshift. However, further similarities between our sample and typical $z>7$ galaxies are based on meager data at high redshifts. These include promising indications that $z>7$ galaxies have hard ionizing spectra (Stark et al. 2015, 2017; Laporte et al. 2017; Mainali et al. 2018) as seen in the LACES sample (Nakajima et al. 2016, 2018), as well as intense [O III] emission characteristic of many high-redshift IRAC-excess sources (Smit et al. 2014, 2015; Roberts-Borsani et al. 2016). Assuming that this is the case, what can be deduced from the fact that $\simeq 20 \%$ of our LACES LAEs meet the canonical criterion for an escape fraction $f_{\text {esc }} \geqslant 10 \%$ required to drive reionization (Robertson et al. 2013, 2015)?

At face value, the average escape fraction for our LACES sample is substantially diminished by the dominant population, for which no significant LyC leakage was detected to quite impressive limits. Nominally the average $f_{\text {esc }}$ would be reduced from $20 \%$ (the mean of the Gold and Silver samples) to only $5 \%$ - a figure in reasonable agreement with the value determined for LBGs by Steidel et al. (2018).
However, if, as seems possible, the dichotomy between our detections and nondetections is largely due to anisotropic LyC leakage, it is likely that the majority of the LACES LAEs are significantly influencing their local IGM. If a similar behavior is present in the reionization era, then such a coarse average would underestimate the role that early equivalents of the LACES population would play in governing reionization. Less luminous versions of our LAEs at high redshift could well have even higher escape fractions, as hinted by the trends in Figure 13. Additionally, the intrinsic fraction of LAEs is observed to increase with redshift (Stark et al. 2010; Schenker et al. 2012), and so we expect that an increasingly large fraction of highredshift star-forming galaxies will look more and more like the LACES sample. Clearly, then, it is crucial to understand physically the dichotomy discussed in Section 6.1.

With this in mind, in later papers we will explore the dependence of $f_{\text {esc }}$ on the ratio of $[\mathrm{O} \mathrm{III]} /[\mathrm{O} \mathrm{III]} \mathrm{to} \mathrm{test} \mathrm{the}$ density-bound concept first promoted by Nakajima \& Ouchi (2014). The present MOSFIRE spectroscopic data have inadequate coverage of [O II] emission, so such correlations cannot yet be examined. In addition, if the dichotomy discussed above originates via anisotropic LyC leakage, numerical simulations suggest that such high escape fractions may arise when feedback creates a turbulent interstellar gas, enabling leakage through porous low-density channels (Kimm \& Cen 2013; Wise et al. 2014). This can be readily tested via IFU spectroscopy, which aims to correlate our HST-determined escape fractions with spatially resolved ISM kinematics.

\section{Summary}

We present the first results from the LymAn Continuum Escape Survey (LACES), where we obtained ultradeep HST WFC3 UVIS/F336W imaging of a sample of 61 faint $z \simeq 3.1$ LAEs and LBGs in the SSA22 field. The extreme depth of the F336W images enabled individual direct detection of escaping Lyman continuum emission $(S / N \geqslant 4)$ in 12 LAEs $(20 \%)$ in our homogeneous sample. Our program provides a huge increase in the number of individually detected LyC leakers at intermediate redshift and represents the first time a large sample of LAEs with a significant fraction of individual leakers has been presented. We make use of extensive multiband photometry, including newly obtained HST WFC3 IR/ F160W imaging, to fit the SED of each galaxy to obtain accurate individual estimates of the escape fraction $f_{\text {esc }}$. We further use our SED-fitting method to infer typical escape fractions from composites of various subsamples. For individual objects we obtain $f_{\text {esc }} \approx 15 \%-60 \%$, and for composites of our Gold and Silver subsamples of Lyman-continuum-detected objects we find $f_{\text {esc }} \geqslant 20 \%$. For our composite of the Lyman continuum nondetection subsample, we infer $f_{\text {esc }} \lesssim 0.5 \%$.

We expect the rate of contamination to be low $(98 \%$ probability $\leqslant 2$ of our detections could be contaminants), and we ensure against foreground interlopers using the high spatial resolution provided by the $\mathrm{F} 336 \mathrm{~W}$ and $\mathrm{F} 160 \mathrm{~W}$ images. We find that the escape fraction may increase for low stellar mass galaxies with larger $\mathrm{EW}_{\mathrm{Ly} \alpha}$.

We discuss the dichotomy between our detections with significant $f_{\text {esc }}$ and our nondetections, seemingly drawn from the same sample, covering the same range of UV luminosities, $\mathrm{EW}_{\mathrm{Ly} \alpha}, \mathrm{EW}_{[\mathrm{O} \mathrm{III}]}$, and $\Delta v_{\mathrm{Ly} \alpha}$. We suggest that this dichotomy could be due to three factors: anisotropic escape where LyC photons escape through channels and the opening angle of these 
channels is only aligned with our line of sight for the detections, a time-varying $f_{\text {esc }}$ due to the bursty nature of star formation in these low-mass systems, or spatially varying $\mathrm{HI}$ in the IGM of the SSA22 protocluster that appears to have a higher $\mathrm{H}$ I density compared to similar control fields (Mawatari et al. 2017).

The large $f_{\text {esc }}$ detected by the LACES program, the fraction of LAEs that leak LyC photons, and the large difference in Lyman continuum flux between detected and nondetected objects may hold significant implications for understanding the mechanisms through which hydrogen-ionizing radiation escapes from galaxies. Coupled with our observations that suggest that low-mass LAEs with strong Ly $\alpha$ have the most extreme $f_{\text {esc }}$, our results provide exciting hints for how to answer the question whether galaxies served as the primary driver of cosmic reionization.

We thank the anonymous referee for helpful comments that improved our manuscript. R.S.E. and T.T. acknowledge funding from the European Research Council (ERC) under the European Union's Horizon 2020 research and innovation programme (grant agreement No. 669253). B.E.R. acknowledges a Maureen and John Hendricks Visiting Professorship at the Institute for Advanced Study, NASA program HST-GO-14747, contract NNG16PJ25C, and grant 17-ATP17-0034. K.N. acknowledges a JSPS Overseas Research Fellowship and a JSPS Research Fellowship for Young Scientists. D.P.S. acknowledges support from the National Science Foundation through grant AST1410155. A.K.I. is supported by the Japan Society for the Promotion of Science, KAKENHI grant No. 17H01114. This work is based on observations taken by the NASA/ESA HST, which is operated by the Association of Universities for Research in Astronomy, Inc., under NASA contract NAS526555. We thank Masato Onodera for observing one of our four MOSFIRE $K$-band masks. We also thank T. Hayashino, T. Yamada, and Y. Matsuda for providing the LAE catalog and the ground-based photometric data. We would also like to thank K. Kakiichi and N. Laporte for useful comments. Further data were taken with the Subaru telescope and the W.M. Keck Observatory on Maunakea, Hawaii, the latter of which is operated as a scientific partnership among the California Institute of Technology, the University of California, and the National Aeronautics and Space Administration. This Observatory was made possible by the generous financial support of the W. M. Keck Foundation. The authors wish to recognize and acknowledge the very significant cultural role and reverence that the summit of Maunakea has always had within the indigenous Hawaiian community. We are most fortunate to have the opportunity to conduct observations from this mountain.

\section{ORCID iDs}

Thomas J. Fletcher (10 https://orcid.org/0000-0002-1633-1117 Brant E. Robertson (iD https://orcid.org/0000-0002-4271-0364 Kimihiko Nakajima (iD https://orcid.org/0000-0003-2965-5070 Richard S. Ellis (i) https://orcid.org/0000-0001-7782-7071 Akio Inoue (i) https://orcid.org/0000-0002-7779-8677

\section{References}

Atek, H., Richard, J., Kneib, J.-P., et al. 2015, ApJ, 800, 18 Barbary, K. 2016, JOSS, 1, 58

Beckwith, S. V. W., Stiavelli, M., Koekemoer, A. M., et al. 2006, AJ, 132, 1729
Behrens, C., Dijkstra, M., \& Niemeyer, J. C. 2014, A\&A, 563, A77 Bertin, E. 2010, Astrophysics Source Code Library, ascl:1010.068 Bertin, E., \& Arnouts, S. 1996, A\&AS, 117, 393

Bian, F., Fan, X., McGreer, I., Cai, Z., \& Jiang, L. 2017, ApJL, 837, L12 Bouwens, R. J., Illingworth, G. D., Oesch, P. A., et al. 2012, ApJL, 752, L5 Bouwens, R. J., Illingworth, G. D., Oesch, P. A., et al. 2015, ApJ, 803, 34 Bouwens, R. J., Smit, R., Labbé, I., et al. 2016, ApJ, 831, 176 Bradley, L. D., Zitrin, A., Coe, D., et al. 2014, ApJ, 792, 76 Calzetti, D., Kinney, A. L., \& Storchi-Bergmann, T. 1994, ApJ, 429, 582 de Barros, S., Vanzella, E., Amorín, R., et al. 2016, A\&A, 585, A51 Dijkstra, M., Gronke, M., \& Venkatesan, A. 2016, ApJ, 828, 71 Eldridge, J., Stanway, E., Xiao, L., et al. 2017, PASA, 34, 58 Eldridge, J. J., \& Stanway, E. R. 2009, MNRAS, 400, 1019

Eldridge, J. J., \& Stanway, E. R. 2012, MNRAS, 419, 479

Ellis, R. S., McLure, R. J., Dunlop, J. S., et al. 2013, ApJL, 763, L7

Faisst, A. L. 2016, ApJ, 829, 99

Feroz, F., \& Hobson, M. P. 2008, MNRAS, 384, 449

Feroz, F., Hobson, M. P., \& Bridges, M. 2009, MNRAS, 398, 1601 Finkelstein, S. L., Papovich, C., Ryan, R. E., et al. 2012, ApJ, 758, 93 Finkelstein, S. L., Ryan, R. E., Jr., Papovich, C., et al. 2015, ApJ, 810, 71 Fontanot, F., Cristiani, S., Pfrommer, C., Cupani, G., \& Vanzella, E. 2014, MNRAS, 438, 2097

Fontanot, F., Cristiani, S., \& Vanzella, E. 2012, MNRAS, 425, 1413 Gordon, K. D., Clayton, G. C., Misselt, K. A., Landolt, A. U., \& Wolff, M. J. 2003, ApJ, 594, 279

Grazian, A., Giallongo, E., Paris, D., et al. 2017, A\&A, 602, A18 Grogin, N. A., Kocevski, D. D., Faber, S. M., et al. 2011, ApJS, 197, 35 Hayashino, T., Inoue, A. K., Kousai, K., et al. 2019, MNRAS, 484, 5868 Hayashino, T., Matsuda, Y., Tamura, H., et al. 2004, AJ, 128, 2073 Hayes, M. 2015, PASA, 32, e027

Illingworth, G. D., Magee, D., Oesch, P. A., et al. 2013, ApJS, 209, 6 Inoue, A. K., \& Iwata, I. 2008, MNRAS, 387, 1681

Inoue, A. K., Iwata, I., \& Deharveng, J.-M. 2006, MNRAS, 371, L1 Inoue, A. K., Iwata, I., Deharveng, J.-M., Buat, V., \& Burgarella, D. 2005, A\&A, 435, 471

Inoue, A. K., Kousai, K., Iwata, I., et al. 2011, MNRAS, 411, 2336 Inoue, A. K., Shimizu, I., Iwata, I., \& Tanaka, M. 2014, MNRAS, 442, 1805 Ishigaki, M., Kawamata, R., Ouchi, M., et al. 2018, ApJ, 854, 73

Iwata, I., Inoue, A. K., Matsuda, Y., et al. 2009, ApJ, 692, 1287 Izotov, Y. I., Orlitová, I., Schaerer, D., et al. 2016a, Natur, 529, 178 Izotov, Y. I., Schaerer, D., Thuan, T. X., et al. 2016b, MNRAS, 461, 3683 Izotov, Y. I., Schaerer, D., Worseck, G., et al. 2018, MNRAS, 474, 4514 Japelj, J., Vanzella, E., Fontanot, F., et al. 2017, MNRAS, 468, 389 Jaskot, A. E., \& Oey, M. S. 2013, ApJ, 766, 91

Kimm, T., \& Cen, R. 2013, ApJ, 776, 35

Kimm, T., Katz, H., Haehnelt, M., et al. 2017, MNRAS, 466, 4826 Koekemoer, A. M., Ellis, R. S., McLure, R. J., et al. 2013, ApJS, 209, 3 Koekemoer, A. M., Faber, S. M., Ferguson, H. C., et al. 2011, ApJS, 197, 36 Kuhlen, M., \& Faucher-Giguère, C.-A. 2012, MNRAS, 423, 862 Labbé, I., Oesch, P. A., Bouwens, R. J., et al. 2013, ApJL, 777, L19 Laporte, N., Nakajima, K., Ellis, R. S., et al. 2017, ApJ, 851, 40 Livermore, R. C., Finkelstein, S. L., \& Lotz, J. M. 2017, ApJ, 835, 113 Lotz, J. M., Koekemoer, A., Coe, D., et al. 2017, ApJ, 837, 97 Ma, X., Hopkins, P. F., Kasen, D., et al. 2016, MNRAS, 459, 3614 Madau, P. 1995, ApJ, 441, 18

Madau, P., \& Haardt, F. 2015, ApJL, 813, L8

Mainali, R., Zitrin, A., Stark, D. P., et al. 2018, MNRAS, 479, 1180

Marchi, F., Pentericci, L., Guaita, L., et al. 2018, A\&A, 614, 11

Matsuda, Y., Yamada, T., Hayashino, T., et al. 2005, ApJL, 634, L125

Matsuda, Y., Yamada, T., Hayashino, T., Yamauchi, R., \& Nakamura, Y. 2006, ApJL, 640, L123

Matthee, J., Sobral, D., Best, P., et al. 2017, MNRAS, 465, 3637

Mawatari, K., Inoue, A. K., Yamada, T., et al. 2017, MNRAS, 467, 3951

McLeod, D. J., McLure, R. J., Dunlop, J. S., et al. 2015, MNRAS, 450, 3032

McLure, R. J., Dunlop, J. S., Bowler, R. A. A., et al. 2013, MNRAS, 432, 2696 Merxlin, E., Bourne, N., Castellano, M., et al. 2016, A\&A, 595, A97 Merxlin, E., Fontana, A., Ferguson, H. C., et al. 2015, A\&A, 582, A15 Micheva, G., Iwata, I., \& Inoue, A. K. 2017a, MNRAS, 465, 302 Micheva, G., Iwata, I., Inoue, A. K., et al. 2017b, MNRAS, 465, 316 Mostardi, R. E., Shapley, A. E., Nestor, D. B., et al. 2013, ApJ, 779, 65 Mostardi, R. E., Shapley, A. E., Steidel, C. C., et al. 2015, ApJ, 810, 107 Naidu, R. P., Forrest, B., Oesch, P. A., Tran, K.-V. H., \& Holden, B. P. 2018, MNRAS, 478, 791

Nakajima, K., Ellis, R. S., Iwata, I., et al. 2016, ApJL, 831, L9

Nakajima, K., Fletcher, T., Ellis, R. S., Robertson, B. E., \& Iwata, I. 2018, MNRAS, 477, 2098 
Nakajima, K., \& Ouchi, M. 2014, MNRAS, 442, 900

Nestor, D. B., Shapley, A. E., Kornei, K. A., Steidel, C. C., \& Siana, B. 2013, ApJ, 765, 47

Nestor, D. B., Shapley, A. E., Steidel, C. C., \& Siana, B. 2011, ApJ, 736, 18

Nonino, M., Dickinson, M., Rosati, P., et al. 2009, ApJS, 183, 244

Oesch, P. A., Bouwens, R. J., Illingworth, G. D., et al. 2013, ApJ, 773, 75

Oesch, P. A., Montes, M., Reddy, N., et al. 2018, ApJS, 237, 12

Oesch, P. A., van Dokkum, P. G., Illingworth, G. D., et al. 2015, ApJL, 804, L30

Oke, J. B., \& Gunn, J. E. 1983, ApJ, 266, 713

Ono, Y., Ouchi, M., Mobasher, B., et al. 2012, ApJ, 744, 83

Ono, Y., Ouchi, M., Shimasaku, K., et al. 2010, MNRAS, 402, 1580

Paardekooper, J.-P., Khochfar, S., \& Dalla Vecchia, C. 2015, MNRAS, 451, 2544

Papovich, C., Dickinson, M., \& Ferguson, H. C. 2001, ApJ, 559, 620

Rafelski, M., Teplitz, H. I., Gardner, J. P., et al. 2015, AJ, 150, 31

Roberts-Borsani, G. W., Bouwens, R. J., Oesch, P. A., et al. 2016, ApJ, 823, 143

Robertson, B. E., Ellis, R. S., Dunlop, J. S., McLure, R. J., \& Stark, D. P. 2010 Natur, 468, 49

Robertson, B. E., Ellis, R. S., Furlanetto, S. R., \& Dunlop, J. S. 2015, ApJL, 802, L19

Robertson, B. E., Furlanetto, S. R., Schneider, E., et al. 2013, ApJ, 768, 71

Rutkowski, M. J., Scarlata, C., Henry, A., et al. 2017, ApJL, 841, L27

Schenker, M. A., Ellis, R. S., Konidaris, N. P., \& Stark, D. P. 2013, ApJ, 777,67

Schenker, M. A., Stark, D. P., Ellis, R. S., et al. 2012, ApJ, 744, 179

Shapley, A. E., Steidel, C. C., Pettini, M., Adelberger, K. L., \& Erb, D. K. 2006, ApJ, 651, 688
Shapley, A. E., Steidel, C. C., Strom, A. L., et al. 2016, ApJL, 826, L24

Shivaei, I., Reddy, N. A., Siana, B., et al. 2018, ApJ, 855, 42

Siana, B., Shapley, A. E., Kulas, K. R., et al. 2015, ApJ, 804, 17

Smit, R., Bouwens, R. J., Franx, M., et al. 2015, ApJ, 801, 122

Smit, R., Bouwens, R. J., Labbé, I., et al. 2014, ApJ, 784, 58

Stanway, E. R., Eldridge, J. J., \& Becker, G. D. 2016, MNRAS, 456, 485

Stark, D. P. 2016, ARA\&A, 54, 761

Stark, D. P., Ellis, R. S., Charlot, S., et al. 2017, MNRAS, 464, 469

Stark, D. P., Ellis, R. S., Chiu, K., Ouchi, M., \& Bunker, A. 2010, MNRAS, 408, 1628

Stark, D. P., Walth, G., Charlot, S., et al. 2015, MNRAS, 454, 1393

Steidel, C. C., Bogosavlevic, M., Shapley, A. E., et al. 2018, ApJ, 869, 123

Steidel, C. C., Pettini, M., \& Adelberger, K. L. 2001, ApJ, 546, 665

Tang, M., Stark, D., Chevallard, J., \& Charlot, S. 2018, arXiv:1809.09637

Trebitsch, M., Blaizot, J., Rosdahl, J., Devriendt, J., \& Slyz, A. 2017, MNRAS, 470,224

Vanzella, E., de Barros, S., Vasei, K., et al. 2016, ApJ, 825, 41

Vanzella, E., Nonino, M., Cupani, G., et al. 2018, MNRAS, 476, L15

Vanzella, E., Siana, B., Cristiani, S., \& Nonino, M. 2010, MNRAS, 404, 1672

Verhamme, A., Orlitová, I., Schaerer, D., et al. 2017, A\&A, 597, A13

Verhamme, A., Orlitová, I., Schaerer, D., \& Hayes, M. 2015, A\&A, 578, A7

Wise, J. H., Demchenko, V. G., Halicek, M. T., et al. 2014, MNRAS, 442, 2560

Yamada, T., Matsuda, Y., Kousai, K., et al. 2012a, ApJ, 751, 29

Yamada, T., Nakamura, Y., Matsuda, Y., et al. 2012b, AJ, 143, 79

Zackrisson, E., Inoue, A. K., \& Jensen, H. 2013, ApJ, 777, 39

Zitrin, A., Labbé, I., Belli, S., et al. 2015, ApJL, 810, L12 